\title{
Global Stability of Positive Periodic Solutions and Almost Periodic Solutions for a Discrete Competitive System
}

\author{
Heping Ma, Jianguo Gao, and Lingling Xie \\ School of Mathematics and Information Science, Beifang University of Nationalities, Yinchuan 750021, China \\ Correspondence should be addressed to Jianguo Gao; gaojguo@163.com
}

Received 18 October 2014; Revised 13 January 2015; Accepted 21 January 2015

Academic Editor: Francisco Solis

Copyright (C) 2015 Heping Ma et al. This is an open access article distributed under the Creative Commons Attribution License, which permits unrestricted use, distribution, and reproduction in any medium, provided the original work is properly cited.

A discrete two-species competitive model is investigated. By using some preliminary lemmas and constructing a Lyapunov function, the existence and uniformly asymptotic stability of positive almost periodic solutions of the system are derived. In addition, an example and numerical simulations are presented to illustrate and substantiate the results of this paper.

\section{Introduction}

Gopalsamy has presented the following Lotka-Volterra competitive system with continuous time version in 1992 (see [1]):

$$
\begin{array}{r}
x_{1}^{\prime}(t)=x_{1}(t)\left[r_{1}(t)-b_{1}(t) x_{1}(t)-\frac{a_{2}(t) x_{2}(t)}{1+d_{1}(t) x_{1}(t)}\right], \\
x_{2}^{\prime}(t)=x_{2}(t)\left[r_{2}(t)-b_{2}(t) x_{2}(t)-\frac{a_{1}(t) x_{1}(t)}{1+d_{2}(t) x_{2}(t)}\right], \\
n=0,1,2, \ldots,
\end{array}
$$

where $x_{1}(t), x_{2}(t)$ represent the population densities of two competing species; $r_{1}(t), r_{2}(t)$ are the intrinsic growth rates of species; $b_{1}(t), b_{2}(t)$ stand for the rates of intraspecific competition of the first and second species, respectively; $a_{2}(t) x_{2}(t) /\left(1+d_{1}(t) x_{1}(t)\right), a_{1}(t) x_{1}(t) /\left(1+d_{2}(t) x_{2}(t)\right)$ denote the competitive response function, respectively. All the coefficients above are continuous and bounded above and below by positive constants.

The discrete-time systems governed by difference equations recently have been won wide-spread attention and applied in studying population growth, the transmission of tuberculosis and HIV/AIDS and influenza prevention and control (see $[2,3]$ ), just because discrete-time models conform better to the reality than the continuous ones, especially for the populations with a short life expectancy or non-overlapping generations. In addition, some works about the bifurcation, chaos, and complex dynamical behaviors of the discrete specie systems have been done (see $[4,5]$ ). In practice, according to the discrete data measured, the discrete-time models commonly provide efficient computational models of continuous models for numerical simulations (see $[2,3,6-11])$. Therefore, we derive the discrete analogue of system (1) by using the same discretization method (see [11]):

$$
\begin{aligned}
& x_{1}(n+1) \\
& =x_{1}(n) \exp \left[r_{1}(n)-b_{1}(n) x_{1}(n)-\frac{a_{2}(n) x_{2}(n)}{1+d_{1}(n) x_{1}(n)}\right], \\
& x_{2}(n+1) \\
& =x_{2}(n) \exp \left[r_{2}(n)-b_{2}(n) x_{2}(n)-\frac{a_{1}(n) x_{1}(n)}{1+d_{2}(n) x_{2}(n)}\right], \\
& n=0,1,2, \ldots,
\end{aligned}
$$

where $x_{i}(n)(i=1,2 ; i \neq j)$ denote the densities of species $x_{i}$ at the $n$th generation, $r_{i}(n)$ stand for the natural growth rates of species $x_{i}$ at the $n$th generation, $b_{i}(n)$ represent the 
self-inhibition rate, respectively, and $a_{j}(n)$ and $d_{i}(n)$ are the interspecific effects of the $n$th generation of species $x_{i}$ on species $x_{j}$.

From an evolutionary perspective, because of the selectivity of species evolution, the periodically varying environments are of vital importance for survival of the fittest. For instance, any periodic change of climate tends to impose its period upon oscillations of internal origin or to cause such oscillations to have a harmonic relation to periodic climatic changes (see [11-16]). Therefore, the coefficients of many systems constructed in ecology are usually considered as periodic functions (see $[12,13]$ ). Not long ago, Wang (see [12]) studied a delayed predator-prey model with HassellVarley type functional responses and obtained the sufficient conditions for the existence of positive periodic solutions by applying the coincidence degree theorem. Many excellent results concerned with the discrete periodic systems are obtained (see [14-16]).

In nature, however, there hardly exists necessarily commensurate periods in the various environment components like seasonal weather change, food supplies, mating habits and harvesting, and so forth. Compared with the periodic systems, we can thus incorporate the assumption of almost periodicity of the coefficients of (1) to reflect the timedependent variability of the environment (see $[6,8-10$, 17]). Recently, Li et al. (see [18]) have proposed an almost periodic discrete predator-prey models with time delays and investigated permanence of the system and the existence of a unique uniformly asymptotically stable positive almost periodic sequence solution. Afterwards, by using Mawhins continuation theorem of the coincidence degree theory, reference [19] achieved some sufficient conditions for the existence of positive almost periodic solutions for a class of delay discrete models with Allee-effect.

Notice that the investigation of periodic solutions and almost periodic solutions is one of the most important topics in the qualitative theory of the difference equations. In this paper, based on the ideas mentioned above, for system (2), one carries out two main works.

(i) Assume that all the coefficients $\left\{r_{i}(n)\right\},\left\{b_{i}(n)\right\}$, $\left\{a_{i}(n)\right\}$, and $\left\{d_{i}(n)\right\}$ are bounded nonnegative periodic sequences. We explore the existence and global stability of positive periodic solutions of system (2) with positive periodic coefficients.

(ii) Furtherly, one discusses the almost periodic solutions of system (2) with positive almost periodic coefficients.

The organization of this paper is as follows. In Section 2 , we present some notations and preliminary lemmas. In Section 3, we seek sufficient conditions which ensure the existence and global stability of positive periodic solutions of system (2). In Section 4, we further investigate the existence, uniqueness, and uniformly asymptotic stability of positive almost periodic solutions for system (2) above. In Section 5 , we present an example and its numerical simulations are carried out to illustrate the feasibility of our main results. In Section 6 , a conclusion is given to conclude this work.

\section{Notations and Preliminaries Lemmas}

Throughout this paper, the notations below will be used:

$$
\begin{aligned}
& h^{u}=\sup _{n \in \mathscr{I}^{+}}\{h(n)\}, \\
& h^{l}=\inf _{n \in \mathscr{I}^{+}}\{h(n)\},
\end{aligned}
$$

where $\{h(n)\}$ is a bounded sequence and $\mathscr{Z}^{+}=\{0,1,2,3, \ldots\}$.

Denote by $\mathscr{R}, \mathscr{R}^{+}, \mathscr{Z}$, and $\mathscr{X}^{+}$the sets of real numbers, nonnegative real numbers, integers, and nonnegative integers, respectively. $\mathscr{R}^{2}$ and $\mathscr{R}^{k}$ are the cones of 2-dimensional and $k$-dimensional real Euclidean spaces, respectively.

Definition 1 (see [10]). A sequence $y: \mathscr{Z} \rightarrow \mathscr{R}^{k}$ is called an almost periodic sequence provided that the following $\varepsilon$ translation set of $y$

$$
I\{\varepsilon, y\}:=\{\tau \in \mathscr{Z}:|y(n+\tau)-y(n)|<\varepsilon, \forall n \in \mathscr{Z}\}
$$

is a relatively dense set in $\mathscr{Z}$ for all $\varepsilon>0$; that is, for any given $\varepsilon>0$, there exists an integer $l(\varepsilon)>0$ such that each discrete interval of length $l(\varepsilon)$ contains a $\tau=\tau(\varepsilon) \in I\{\varepsilon, y\}$ such that

$$
|y(n+\tau)-y(n)|<\varepsilon, \quad \forall n \in \mathscr{Z} ;
$$

$\tau$ is referred to as the $\varepsilon$-translation number of $y(n)$.

Definition 2 (see [10]). Suppose $h: \mathscr{Z} \times \mathscr{A} \rightarrow R^{k}$, where $\mathscr{A}$ is an open set in $\mathscr{R}^{k} \cdot h(n, x)$ is said to be almost periodic in $n$ uniformly for $x \in \mathscr{A}$ or uniformly almost periodic for short, if for any $\varepsilon>0$ and any compact set $\mathcal{S}$ in $\mathscr{A}$ there exists a positive integer $l(\varepsilon, \mathcal{S})$ such that any interval of length $l(\varepsilon, \mathcal{S})$ contains an integer $\tau$ for which

$$
|h(n+\tau, x)-h(n, x)|<\varepsilon, \quad \forall n \in \mathscr{Z}, x \in \mathcal{S} ;
$$

$\tau$ is called the $\varepsilon$-translation number of $h(n, x)$.

Lemma 3 (see [10]). $\{x(n)\}$ is an almost periodic sequence if and only if for any sequence $\left\{p_{t}^{\prime}\right\} \subset \mathscr{Z}$ there exists a subsequence $\left\{p_{t}\right\} \subset\left\{p_{t}^{\prime}\right\}$ such that $x\left(n+p_{t}\right)$ converges uniformly on $n \in \mathscr{Z}$ ast $\rightarrow \infty$. Thus, the limit sequence is also an almost periodic sequence.

Furthermore, we consider the following almost periodic difference system:

$$
x(n+1)=h(n, x(n)), \quad n \in \mathscr{Z}^{+},
$$

where $h: \mathscr{Z}^{+} \times \mathscr{C}_{B} \rightarrow \mathscr{R}^{k}, \mathscr{C}_{B}=\{x \in \mathscr{C}:\|x\|<B\}$, and $h(n, x)$ is almost periodic in $n$ uniformly for $x \in \mathscr{C}_{B}$ and is continuous in $x$.

The product system of (7) is in the following form:

$$
\begin{aligned}
& x(n+1)=h\left(n, x_{n}\right), \\
& y(n+1)=h\left(n, y_{n}\right),
\end{aligned}
$$

and [20] obtained the following lemma, where $(x(n, \phi), y(n$, $\psi)$ ) is a solution of (8). 
Lemma 4 (see [20]). Suppose there exists a Lyapunov function $V(n, \phi, \psi)$ defined for $n \in \mathscr{Z}^{+},\|\phi\|<B$, and $\|\psi\|<B$ satisfying the following conditions:

(1) $\alpha(|\phi-\psi|) \leq V(n, \phi, \psi) \leq \beta(\|\phi-\psi\|)$, where $\alpha, \beta \in p$ with $p=\{\eta:[0, \infty) \rightarrow[0, \infty) \mid \eta(0)=0$ and $\eta(\nu)$ is continuous, increasing in $\nu$;

(2) $\left|V\left(n, \phi_{1}, \psi_{1}\right)-V\left(n, \phi_{2}, \psi_{2}\right)\right| \leq L\left(\left\|\phi_{1}-\phi_{2}\right\|+\left\|\psi_{1}-\psi_{2}\right\|\right)$, where $L>0$ is a constant;

(3) $\Delta V_{(8)}(n, \phi, \psi) \leq-\gamma V(n, \phi, \psi)$, where $0<\gamma<$ 1 is a constant and $\Delta V_{(8)}(n, \phi, \psi)=V(n+$ $\left.1, x_{n+1}(n, \phi), y_{n+1}(n, \psi)\right)-V(n, \phi, \psi)$.

Moreover, suppose that there exists a solution $x(n)$ of system (7) such that $\left\|x_{n}\right\| \leq B^{*}<B$ for all $n \in \mathscr{Z}^{+}$; then there exists a unique uniformly asymptotically stable almost periodic solution $q(n)$ of system (7) which satisfies $|q(n)| \leq B^{*}$. In particular, if $h(n, \phi)$ is periodic of period $\omega$, then system (7) has a unique uniformly asymptotically stable periodic solution with $\operatorname{period} \omega$.

Lemma 5 (see [21]). Any positive solution $\left(x_{1}(n), x_{2}(n)\right)$ of system (2) satisfies

$$
\lim \sup _{n \rightarrow+\infty} x_{i}(n) \leq \frac{\exp \left(r_{i}^{u}-1\right)}{b_{i}^{l}} \equiv M_{i}, \quad i=1,2 .
$$

Lemma 6 (see [21]). Let system (2) satisfy the following assumptions:

$$
\min \left\{r_{1}^{l}-a_{2}^{u} M_{2}, r_{2}^{l}-a_{1}^{u} M_{1}\right\}>0 .
$$

Then, any positive solution $\left(x_{1}(n), x_{2}(n)\right)$ of system (2) satisfies

$$
\begin{array}{r}
x_{i}(n) \geq \frac{r_{i}^{l}-a_{j}^{u} M_{j}}{b_{i}^{u}} \exp \left(r_{i}^{l}-a_{j}^{u} M_{j}-b_{i}^{u} M_{i}\right) \equiv m_{i}, \\
i \neq j ; \quad i, j=1,2 .
\end{array}
$$

\section{Existence and Stability of Positive Periodic Solutions}

Apparently, the permanence of system (2) can be obtained according to Lemmas 5 and 6 . In the following, we will show the existence and stability of positive periodic solutions of system (2). To this end, let us assume that all the coefficients of system (2) are $\delta$-periodic; namely,

$$
\begin{array}{r}
r_{i}(n+\delta)=r_{i}(n), \\
a_{i}(n+\delta)=a_{i}(n), \\
b_{i}(n+\delta)=b_{i}(n), \\
d_{i}(n+\delta)=d_{i}(n), \\
i=1,2 .
\end{array}
$$

Lemma 7 (see [16]). If the assumption (10) holds, then system (2) has at least one strictly positive $\delta$-periodic solution and is denoted by $\left(x_{1}^{*}(n), x_{2}^{*}(n)\right)$.
Definition 8. A positive periodic solution $\left(x_{1}^{*}(n), x_{2}^{*}(n)\right)$ of system (2) is globally stable if each other solution $\left(x_{1}(n), x_{2}(n)\right)$ with positive initial value defined for all $n>0$ satisfies

$$
\begin{aligned}
& \lim _{n \rightarrow+\infty}\left|x_{1}(n)-x_{1}^{*}(n)\right|=0, \\
& \lim _{n \rightarrow+\infty}\left|x_{2}(n)-x_{2}^{*}(n)\right|=0 .
\end{aligned}
$$

Now, we present the main results.

Theorem 9. Let the following assumption

$$
\begin{array}{r}
\xi_{1}=\max \left\{\left|1+a_{2}^{u} d_{1}^{u} M_{1} M_{2}-b_{1}^{l} m_{1}\right|,\right. \\
\left.\left|1+a_{2}^{l} d_{1}^{l} m_{1} m_{2}-b_{1}^{u} M_{1}\right|\right\} \\
+a_{2}^{u} M_{2}+a_{2}^{u} d_{1}^{u} M_{1} M_{2}<1, \\
\xi_{2}=\max \left\{\left|1+a_{1}^{u} d_{2}^{u} M_{1} M_{2}-b_{2}^{l} m_{2}\right|,\right. \\
\left.\left|1+a_{1}^{l} d_{2}^{l} m_{1} m_{2}-b_{2}^{u} M_{2}\right|\right\} \\
+a_{1}^{u} M_{1}+a_{1}^{u} d_{2}^{u} M_{1} M_{2}<1
\end{array}
$$

and (10) hold; then the positive periodic solution of system (2) is globally stable.

Proof. Let $\left(x_{1}^{*}(n), x_{2}^{*}(n)\right)$ be a positive periodic solution of system (2).

Denote $\exp z_{1}(n)=x_{1}(n) / x_{1}^{*}(n)$ and $\exp z_{2}(n)=x_{2}(n) /$ $x_{2}^{*}(n)$; then we have

$$
\begin{aligned}
z_{1}(n+1) & \\
= & z_{1}(n)+b_{1}(n) x_{1}^{*}(n)\left(1-\exp z_{1}(n)\right) \\
& +\left(a_{2}(n)\left(x_{2}^{*}(n)-x_{2}(n)\right)\right. \\
& \left.\quad+a_{2}(n) d_{1}(n)\left(x_{1}(n) x_{2}^{*}(n)-x_{1}^{*}(n) x_{2}(n)\right)\right) \\
& \cdot\left(\left(1+d_{1}(n) x_{1}^{*}(n)\right)\left(1+d_{1}(n) x_{1}(n)\right)\right)^{-1} \\
\leq & z_{1}(n)+b_{1}(n) x_{1}^{*}(n)\left(1-\exp z_{1}(n)\right) \\
& +a_{2}(n) x_{2}^{*}(n)\left(1-\exp z_{2}(n)\right) \\
& +a_{2}(n) d_{1}(n) x_{1}^{*}(n) x_{2}^{*}(n) \\
& \cdot\left[\left(1-\exp z_{2}(n)\right)-\left(1-\exp z_{1}(n)\right)\right], \\
z_{2}(n+1) & \\
= & z_{2}(n)+b_{2}(n) x_{2}^{*}(n)\left(1-\exp z_{2}(n)\right) \\
+ & \left(a_{1}(n)\left(x_{1}^{*}(n)-x_{1}(n)\right)\right. \\
& \left.+a_{1}(n) d_{2}(n)\left(x_{1}^{*}(n) x_{2}(n)-x_{1}(n) x_{2}^{*}(n)\right)\right) \\
& \left(\left(1+d_{2}(n) x_{2}^{*}(n)\right)\left(1+d_{2}(n) x_{2}(n)\right)\right)^{-1}
\end{aligned}
$$




$$
\begin{aligned}
\leq & z_{2}(n)+b_{2}(n) x_{2}^{*}(n)\left(1-\exp z_{2}(n)\right) \\
& +a_{1}(n) x_{1}^{*}(n)\left(1-\exp z_{1}(n)\right) \\
& +a_{1}(n) d_{2}(n) x_{1}^{*}(n) x_{2}^{*}(n) \\
& \cdot\left[\left(1-\exp z_{1}(n)\right)-\left(1-\exp z_{2}(n)\right)\right],
\end{aligned}
$$

which, according to the mean value, yields

$$
\begin{aligned}
& z_{1}(n+1) \\
& \leq z_{1}(n)\left[1-b_{1}(n) x_{1}^{*}(n) \exp \left(\vartheta_{1} z_{1}(n)\right)\right. \\
& \left.+a_{2}(n) d_{1}(n) x_{1}^{*}(n) x_{2}^{*}(n) \exp \left(\vartheta_{1} z_{1}(n)\right)\right] \\
& -z_{2}(n)\left[a_{2}(n) x_{2}^{*}(n) \exp \left(\vartheta_{2} z_{2}(n)\right)\right. \\
& \left.+a_{2}(n) d_{1}(n) x_{1}^{*}(n) x_{2}^{*}(n) \exp \left(\vartheta_{2} z_{2}(n)\right)\right] \\
& z_{2}(n+1) \quad \\
& \leq z_{2}(n)\left[1-b_{2}(n) x_{2}^{*}(n) \exp \left(\vartheta_{3} z_{2}(n)\right)\right. \\
& \left.+a_{1}(n) d_{2}(n) x_{1}^{*}(n) x_{2}^{*}(n) \exp \left(\vartheta_{3} z_{2}(n)\right)\right] \\
& -z_{1}(n)\left[a_{1}(n) x_{1}^{*}(n) \exp \left(\vartheta_{4} z_{1}(n)\right)\right. \\
& \left.+a_{1}(n) d_{2}(n) x_{1}^{*}(n) x_{2}^{*}(n) \exp \left(\vartheta_{4} z_{1}(n)\right)\right]
\end{aligned}
$$

where all the constants $\vartheta_{1}, \vartheta_{2}, \vartheta_{3}, \vartheta_{4} \in(0,1)$. Obviously, together with (14) we can find a sufficiently small $\varepsilon$ such that

$$
\begin{aligned}
\xi_{1}^{*}= & \max \left\{\left|1+a_{2}^{u} d_{1}^{u}\left(M_{1}+\varepsilon\right)\left(M_{2}+\varepsilon\right)-b_{1}^{l}\left(m_{1}-\varepsilon\right)\right|,\right. \\
& \left.\left|1+a_{2}^{l} d_{1}^{l}\left(m_{1}-\varepsilon\right)\left(m_{2}-\varepsilon\right)-b_{1}^{u}\left(M_{1}+\varepsilon\right)\right|\right\} \\
& +a_{2}^{u}\left(M_{2}+\varepsilon\right)+a_{2}^{u} d_{1}^{u}\left(M_{1}+\varepsilon\right)\left(M_{2}+\varepsilon\right)<1 \\
\xi_{2}^{*}= & \max \left\{\left|1+a_{1}^{u} d_{2}^{u}\left(M_{1}+\varepsilon\right)\left(M_{2}+\varepsilon\right)-b_{2}^{l}\left(m_{2}-\varepsilon\right)\right|,\right. \\
& \left.\left|1+a_{1}^{l} d_{2}^{l}\left(m_{1}-\varepsilon\right)\left(m_{2}-\varepsilon\right)-b_{2}^{u}\left(M_{2}+\varepsilon\right)\right|\right\} \\
& +a_{1}^{u}\left(M_{1}+\varepsilon\right)+a_{1}^{u} d_{2}^{u}\left(M_{1}+\varepsilon\right)\left(M_{2}+\varepsilon\right)<1
\end{aligned}
$$

It follows from Lemmas 5 and 6 that there exists an $N_{0}$ such that $n>N_{0}$; we have

$$
\begin{aligned}
& 0<m_{1}-\varepsilon \leq x_{1}^{*}(n) \leq M_{1}+\varepsilon \\
& 0<m_{2}-\varepsilon \leq x_{2}^{*}(n) \leq M_{2}+\varepsilon \\
& 0<m_{1}-\varepsilon \leq x_{1}(n) \leq M_{1}+\varepsilon \\
& 0<m_{2}-\varepsilon \leq x_{2}(n) \leq M_{2}+\varepsilon
\end{aligned}
$$

Then one obtains the fact that both $x_{1}^{*}(n) \exp \left(\vartheta_{1} z_{1}(n)\right)$ and $x_{1}^{*}(n) \exp \left(\vartheta_{4} z_{1}(n)\right)$ are between $x_{1}^{*}(n)$ and $x_{1}(n)$. Similarly, both $x_{2}^{*}(n) \exp \left(\vartheta_{2} z_{2}(n)\right)$ and $x_{2}^{*}(n) \exp \left(\vartheta_{3} z_{2}(n)\right)$ are between $x_{2}^{*}(n)$ and $x_{2}(n)$. From the first equation of (2), one has

$$
\begin{aligned}
& \left|z_{1}(n+1)\right| \\
& \leq\left|z_{1}(n)\right| \mid\left[1-b_{1}(n) x_{1}^{*}(n) \exp \left(\vartheta_{1} z_{1}(n)\right)\right. \\
& \left.+a_{2}(n) d_{1}(n) x_{1}^{*}(n) x_{2}^{*}(n) \exp \left(\vartheta_{1} z_{1}(n)\right)\right] \mid \\
& +\left|z_{2}(n)\right| \mid\left[a_{2}(n) x_{2}^{*}(n) \exp \left(\vartheta_{2} z_{2}(n)\right)\right. \\
& \left.+a_{2}(n) d_{1}(n) x_{1}^{*}(n) x_{2}^{*}(n) \exp \left(\vartheta_{2} z_{2}(n)\right)\right] \mid \\
& \leq \max \left\{\left|1+a_{2}^{u} d_{1}^{u}\left(M_{1}+\varepsilon\right)\left(M_{2}+\varepsilon\right)-b_{1}^{l}\left(m_{1}-\varepsilon\right)\right|,\right. \\
& \left.\left|1+a_{2}^{l} d_{1}^{l}\left(m_{1}-\varepsilon\right)\left(m_{2}-\varepsilon\right)-b_{1}^{u}\left(M_{1}+\varepsilon\right)\right|\right\} \\
& \cdot\left|z_{1}(n)\right| \\
& +\left[a_{2}^{u}\left(M_{2}+\varepsilon\right)+a_{2}^{u} d_{1}^{u}\left(M_{1}+\varepsilon\right)\left(M_{2}+\varepsilon\right)\right]\left|z_{2}(n)\right| \\
& \leq \xi_{1}^{*} \max \left\{z_{1}(n), z_{2}(n)\right\} \text {. }
\end{aligned}
$$

Similar to the arguments as above, we must have

$$
\left|z_{2}(n+1)\right| \leq \xi_{2}^{*} \max \left\{z_{1}(n), z_{2}(n)\right\} .
$$

We denote $\xi^{*}=\max \left\{x_{1}^{*}, x_{2}^{*}\right\}$; then $\xi^{*}<1$. Therefore, provided that $n>N_{0}$,

$$
\begin{aligned}
& \max \left\{\left|z_{1}(n+1)\right|,\left|z_{2}(n+1)\right|\right\} \\
& \quad \leq \xi^{*} \max \left\{\left|z_{1}(n)\right|,\left|z_{2}(n)\right|\right\} \leq\left(\xi^{*}\right)^{n-N_{0}} .
\end{aligned}
$$

Consequently, $\lim _{n \rightarrow+\infty}\left|x_{i}(n)-x_{i}^{*}(n)\right|=0$, where $i=1,2$. By Definition 8, it follows that the positive periodic solution $\left\{x_{1}^{*}(n), x_{2}^{*}(n)\right\}$ of system (2) is globally stable. This completes the proof.

\section{Existence and Stability of Positive Almost Periodic Solutions}

In this section, we discuss the existence of positive almost periodic solutions of system (2).

Lemma 10. If assumption (10) is true, then $\Omega \neq \varnothing$.

Proof. According to an inductive argument, system (2) is actually described as follows:

$$
\begin{aligned}
& x_{1}(n) \\
& =x_{1}(0) \exp \sum_{t=0}^{n-1}\left[r_{1}(t)-b_{1}(t) x_{1}(t)-\frac{a_{2}(t) x_{2}(t)}{1+d_{1}(t) x_{1}(t)}\right], \\
& x_{2}(n) \\
& =x_{2}(0) \exp \sum_{t=0}^{n-1}\left[r_{2}(t)-b_{2}(t) x_{2}(t)-\frac{a_{1}(t) x_{1}(t)}{1+d_{2}(t) x_{2}(t)}\right] .
\end{aligned}
$$


Combining Lemmas 5 and 6, for any solution $\left(x_{1}(n), x_{2}(n)\right)$ of system (2) and an arbitrarily small constant $\varepsilon>0$, there must be $n_{0}$ which is sufficiently large such that

$$
\begin{array}{r}
m_{1}-\varepsilon \leq x_{1}(n) \leq M_{1}+\varepsilon \\
m_{2}-\varepsilon \leq x_{2}(n) \leq M_{2}+\varepsilon \\
\forall n \geq n_{0} .
\end{array}
$$

Assuming that $\tau_{k}$ is any positive integer sequence such that $\left\{\tau_{k}\right\} \rightarrow+\infty$ as $k \rightarrow+\infty$, we can prove that there is a subsequence of $\left\{\tau_{k}\right\}$ still denoted by $\left\{\tau_{k}\right\}$, such that $x_{i}(n+$ $\left.\tau_{k}\right) \rightarrow x_{i}^{*}(n), i=1,2$ uniformly in $n$ on any finite subset $L$ of $\mathscr{Z}^{+}$as $k \rightarrow+\infty$, where $L=\left\{l_{1}, l_{2}, \ldots, l_{m}\right\}, l_{j} \in \mathscr{Z}^{+}(j=$ $1,2, \ldots, m)$, and $m$ is a finite number.

In fact, for any finite subset $L \subset \mathscr{Z}^{+}, \tau_{k}+l_{j}>n_{0},(j=$ $1,2, \ldots, m)$, when $k$ is large enough. Therefore, $m_{i}-\varepsilon \leq x_{i}(n+$ $\left.\tau_{k}\right) \leq M_{i}-\varepsilon, i=1,2$; that is, $x_{i}\left(n+\tau_{k}\right)$ are uniformly bounded when $k$ is sufficiently large.

Next, for $l_{1} \in L$, we choose a subsequence $\left\{\tau_{k}^{(1)}\right\}$ of $\left\{\tau_{k}\right\}$ such that $x_{1}\left(l_{1}+\tau_{k}^{(1)}\right)$ and $x_{2}\left(l_{1}+\tau_{k}^{(1)}\right)$ uniformly converge on $\mathscr{Z}^{+}$for $k$ sufficient large.

Similar to the arguments of $l_{1}$, for $l_{2} \in L$, one can select a subsequence $\left\{\tau_{k}^{(2)}\right\}$ of $\left\{\tau_{k}^{(1)}\right\}$ such that $x_{1}\left(l_{2}+\tau_{k}^{(2)}\right)$ and $x_{2}\left(l_{2}+\right.$ $\tau_{k}^{(2)}$ ) uniformly converge on $\mathscr{Z}^{+}$for large enough $k$.

Repeating above-mentioned process, for $l_{m} \in L$, one obtains a subsequence $\left\{\tau_{k}^{(m)}\right\}$ of $\left\{\tau_{k}^{(m-1)}\right\}$ such that $x_{1}\left(l_{m}+\tau_{k}^{(m)}\right)$ and $x_{2}\left(l_{m}+\tau_{k}^{(m)}\right)$ uniformly converge on $\mathscr{Z}^{+}$for sufficiently large $k$.

Based on the above, one selects the sequence $\left\{\tau_{k}^{(m)}\right\}$ which is a subsequence of $\left\{\tau_{k}\right\}$ still denoted by $\left\{\tau_{k}\right\}$; then, for $n \in$ $L$, one gets $x_{i}\left(n+\tau_{k}\right) \rightarrow x_{i}^{*}(n), i=1,2$ uniformly in $n \in$ $L$ as $k \rightarrow+\infty$. So the conclusion holds truely due to the arbitrariness of $L$.

Different from Section 3, we suppose that all the coefficients $\left\{r_{i}(n)\right\},\left\{a_{i}(n)\right\},\left\{b_{i}(n)\right\}$, and $\left\{d_{i}(n)\right\}, i=1,2$, are bounded nonnegative almost periodic sequences; for the above sequence $\left\{\tau_{k}\right\}, \tau_{k} \rightarrow+\infty$ as $k \rightarrow+\infty$, there exists a subsequence denoted by $\left\{\tau_{k}\right\}$ such that

$$
\begin{array}{r}
r_{i}\left(n+\tau_{k}\right) \longrightarrow r_{i}(n), \\
b_{i}\left(n+\tau_{k}\right) \longrightarrow b_{i}(n), \\
a_{i}\left(n+\tau_{k}\right) \longrightarrow a_{i}(n), \\
d_{i}\left(n+\tau_{k}\right) \longrightarrow d_{i}(n), \\
i=1,2,
\end{array}
$$

as $k \rightarrow+\infty$ uniformly on $\mathscr{Z}^{+}$.

For any $\rho \in \mathscr{Z}^{+}$, assume that $\tau_{k}+\rho \geq N_{0}$ when $k$ is large enough. By an inductive argument of system (2) from $\tau_{k}+\rho$ to $n+\tau_{k}+\rho$, where $n \in \mathscr{Z}^{+}$, one obtains

$$
\begin{aligned}
& x_{1}\left(n+\tau_{k}+\rho\right) \\
& =x_{1}\left(\tau_{k}+\rho\right)
\end{aligned}
$$

$$
\begin{aligned}
& \cdot \exp \sum_{t=\tau_{k}+\rho}^{n+\tau_{k}+\rho-1}\left[r_{1}(t)-b_{1}(t) x_{1}(t)-\frac{a_{2}(t) x_{2}(t)}{1+d_{1}(t) x_{1}(t)}\right], \\
& x_{2}\left(n+\tau_{k}+\rho\right) \\
& =x_{2}\left(\tau_{k}+\rho\right) \\
& \cdot \exp \sum_{t=\tau_{k}+\rho}^{n+\tau_{k}+\rho-1}\left[r_{2}(t)-b_{2}(t) x_{2}(t)-\frac{a_{1}(t) x_{1}(t)}{1+d_{2}(t) x_{2}(t)}\right] .
\end{aligned}
$$

Hence, (25) yields

$$
\begin{aligned}
& x_{1}\left(n+\tau_{k}+\rho\right) \\
& =x_{1}\left(\tau_{k}+\rho\right) \\
& \quad \cdot \exp \sum_{t=\rho}^{n+\rho-1}\left[r_{1}\left(t+\tau_{k}\right)-b_{1}\left(t+\tau_{k}\right) x_{1}\left(t+\tau_{k}\right)\right. \\
& \left.x_{2}\left(n+\tau_{k}+\rho\right) \quad-\frac{a_{2}\left(t+\tau_{k}\right) x_{2}\left(t+\tau_{k}\right)}{1+d_{1}\left(t+\tau_{k}\right) x_{1}\left(t+\tau_{k}\right)}\right], \\
& =x_{2}\left(\tau_{k}+\rho\right) \\
& \cdot \exp \sum_{t=\rho}^{n+\rho-1}\left[r_{2}\left(t+\tau_{k}\right)-b_{2}\left(t+\tau_{k}\right) x_{2}\left(t+\tau_{k}\right)\right. \\
& \left.-\frac{a_{1}\left(t+\tau_{k}\right) x_{1}\left(t+\tau_{k}\right)}{1+d_{2}\left(t+\tau_{k}\right) x_{2}\left(t+\tau_{k}\right)}\right] .
\end{aligned}
$$

Let $k \rightarrow+\infty$; one has

$$
\begin{aligned}
& x_{1}^{*}(n+\rho) \\
& =x_{1}^{*}(\rho) \\
& \quad \cdot \exp \sum_{t=\rho}^{n+\rho-1}\left[r_{1}(t)-b_{1}(t) x_{1}^{*}(t)-\frac{a_{2}(t) x_{2}^{*}(t)}{1+d_{1}(t) x_{1}^{*}(t)}\right], \\
& x_{2}^{*}(n+\rho) \\
& =x_{2}^{*}(\rho) \\
& \quad \cdot \exp \sum_{t=\rho}^{n+\rho-1}\left[r_{2}(t)-b_{2}(t) x_{2}^{*}(t)-\frac{a_{1}(t) x_{1}^{*}(t)}{1+d_{2}(t) x_{2}^{*}(t)}\right] .
\end{aligned}
$$

It is easy to see that $\left(x_{1}^{*}(n), x_{2}^{*}(n)\right)$ is a solution of system (2) on $\mathscr{Z}^{+}$for arbitrary $\rho$, and

$$
\begin{array}{r}
0<m_{1}-\varepsilon \leq x_{1}^{*}(n) \leq M_{1}-\varepsilon, \\
0<m_{2}-\varepsilon \leq x_{2}^{*}(n) \leq M_{2}-\varepsilon, \\
n \in \mathscr{Z}^{+} .
\end{array}
$$


Then we get (29) due to $\varepsilon$ which is an arbitrarily small positive constant:

$$
\begin{array}{r}
0<m_{1} \leq x_{1}^{*}(n) \leq M_{1}, \\
0<m_{2} \leq x_{2}^{*}(n) \leq M_{2}, \\
n \in \mathscr{Z}^{+} .
\end{array}
$$

This completes the proof.

Finally, we are ready to state our main result in this section.

Theorem 11. Let the assumption (10) be satisfied and the following statement in which $0<\gamma<1$ holds true, where $\gamma=\min \left\{\beta_{1}, \beta_{2}\right\}$ :

$$
\begin{aligned}
& \beta_{1}=2 b_{1}^{l} m_{1}-\frac{a_{2}^{u} M_{2}+a_{2}^{u} b_{1}^{u} M_{1} M_{2}}{1+d_{1}^{l} m_{1}} \\
& -\frac{a_{1}^{u} M_{1}+a_{1}^{u} b_{2}^{u} M_{1} M_{2}}{1+d_{2}^{l} m_{2}}-\left(b_{1}^{u} M_{1}\right)^{2} \\
& -\frac{2\left(a_{2}^{u} d_{1}^{u} M_{1} M_{2}-a_{2}^{l} b_{1}^{l} d_{1}^{l} m_{1}^{2} m_{2}\right)}{\left(1+d_{1}^{l} m_{1}\right)^{2}} \\
& -\frac{\left(a_{1}^{u} M_{1}\right)^{2}}{\left(1+d_{2}^{l} m_{2}\right)^{2}}-\frac{a_{2}^{u 2} d_{1}^{u} M_{1} M_{2}^{2}}{\left(1+d_{1}^{l} m_{1}\right)^{3}}-\frac{a_{1}^{u 2} d_{2}^{u} M_{1}^{2} M_{2}}{\left(1+d_{2}^{l} m_{2}\right)^{3}} \\
& -\frac{\left(a_{2}^{u} d_{1}^{u} M_{1} M_{2}\right)^{2}}{\left(1+d_{1}^{l} m_{1}\right)^{4}} \\
& \beta_{2}=2 b_{2}^{l} m_{2}-\frac{a_{2}^{u} M_{2}+a_{2}^{u} b_{1}^{u} M_{1} M_{2}}{1+d_{1}^{l} m_{1}} \\
& -\frac{a_{1}^{u} M_{1}+a_{1}^{u} b_{2}^{u} M_{1} M_{2}}{1+d_{2}^{l} m_{2}}-\left(b_{2}^{u} M_{2}\right)^{2} \\
& -\frac{2\left(a_{1}^{u} d_{2}^{u} M_{1} M_{2}-a_{2}^{l} b_{2}^{l} d_{2}^{l} m_{1} m_{2}^{2}\right)}{\left(1+d_{2}^{l} m_{2}\right)^{2}} \\
& -\frac{\left(a_{2}^{u} M_{2}\right)^{2}}{\left(1+d_{1}^{l} m_{1}\right)^{2}}-\frac{a_{2}^{u 2} d_{1}^{u} M_{1} M_{2}^{2}}{\left(1+d_{1}^{l} m_{1}\right)^{3}}-\frac{a_{1}^{u 2} d_{2}^{u} M_{1}^{2} M_{2}}{\left(1+d_{2}^{l} m_{2}\right)^{3}} \\
& -\frac{\left(a_{1}^{u} d_{2}^{u} M_{1} M_{2}\right)^{2}}{\left(1+d_{2}^{l} m_{2}\right)^{4}}
\end{aligned}
$$

then system (2) admits a unique positive almost periodic solution, which is uniformly asymptotically stable.

Proof. Denote $u_{1}(n)=\ln x_{1}(n)$ and $u_{2}(n)=\ln x_{2}(n)$. It follows from (2) that

$$
\begin{gathered}
u_{1}(n+1)=u_{1}(n)+r_{1}(n)-b_{1}(n) e^{u_{1}(n)} \\
-\frac{a_{2}(n) e^{u_{2}(n)}}{1+d_{1}(n) e^{u_{1}(n)}}
\end{gathered}
$$

$$
\begin{aligned}
u_{2}(n+1)= & u_{2}(n)+r_{2}(n)-b_{2}(n) e^{u_{2}(n)} \\
& -\frac{a_{1}(n) e^{u_{1}(n)}}{1+d_{2}(n) e^{u_{2}(n)}} .
\end{aligned}
$$

By Lemma 10, it is easy to see that for system (31) there exists a bounded solution $\left(u_{1}(n), u_{2}(n)\right)$ satisfying

$$
\begin{array}{r}
\ln m_{1} \leq u_{1}(n) \leq \ln M_{1}, \\
\ln m_{2} \leq u_{2}(n) \leq \ln M_{2}, \\
n \in \mathscr{Z}^{+} .
\end{array}
$$

Thus $\left|u_{1}(n)\right| \leq s_{1}$ and $\left|u_{2}(n)\right| \leq s_{2}$, where $s_{1}=\max \left\{\left|\ln M_{1}\right|\right.$, $\left.\left|\ln m_{1}\right|\right\}$ and $s_{2}=\max \left\{\left|\ln M_{2}\right|,\left|\ln m_{2}\right|\right\}$. Define the norm:

$$
\left\|\left(u_{1}(n), u_{2}(n)\right)\right\|=\left|u_{1}(n)\right|+\left|u_{2}(n)\right|
$$

where $\left(u_{1}(n), u_{2}(n)\right) \in \mathscr{R}^{2}$.

Consider the product system of system (31) as follows:

$$
\begin{aligned}
u_{1}(n+1)= & u_{1}(n)+r_{1}(n)-b_{1}(n) e^{u_{1}(n)} \\
& -\frac{a_{2}(n) e^{u_{2}(n)}}{1+d_{1}(n) e^{u_{1}(n)}}, \\
u_{2}(n+1)= & u_{2}(n)+r_{2}(n)-b_{2}(n) e^{u_{2}(n)} \\
& -\frac{a_{1}(n) e^{u_{1}(n)}}{1+d_{2}(n) e^{u_{2}(n)}}, \\
w_{1}(n+1)= & w_{1}(n)+r_{1}(n)-b_{1}(n) e^{w_{1}(n)} \\
& -\frac{a_{2}(n) e^{w_{2}(n)}}{1+d_{1}(n) e^{w_{1}(n)}}, \\
w_{2}(n+1)= & w_{2}(n)+r_{2}(n)-b_{2}(n) e^{w_{2}(n)} \\
& -\frac{a_{1}(n) e^{w_{1}(n)}}{1+d_{2}(n) e^{w_{2}(n)}} .
\end{aligned}
$$

Assume that $H=\left(u_{1}(n), u_{2}(n)\right)$ and $I=\left(w_{1}(n), w_{2}(n)\right)$ are any two solutions of system (18) defined on $\Gamma$. And then $\|H\| \leq$ $S$ and $\|I\| \leq S$, where $S=s_{1}+s_{2}$, and $\Gamma=\left\{\left(u_{1}(n), u_{2}(n)\right) \mid\right.$ $\left.\ln m_{i} \leq u_{i}(n) \leq \ln M_{i}, i=1,2, n \in \mathscr{Z}^{+}\right\}$.

In the following, construct a Lyapunov function which is defined on $\mathscr{Z}^{+} \times \Gamma \times \Gamma$ :

$$
V(n, H, I)=\left(u_{1}(n)-w_{1}(n)\right)^{2}+\left(u_{2}(n)-w_{2}(n)\right)^{2} .
$$

Notice that the form $\|H-I\|=\left|u_{1}(n)-w_{1}(n)\right|+\mid u_{2}(n)-$ $w_{2}(n) \mid$ is equivalent to $\|H-I\|_{\Delta}=\left[\left(u_{1}(n)-w_{1}(n)\right)^{2}+\right.$ $\left.\left(u_{2}(n)-w_{2}(n)\right)^{2}\right]^{1 / 2}$, which implies that there exist two positive constants $K_{1}, K_{2}$ such that $K_{1}\|H-I\| \leq\|H-I\| \leq$ $K_{2}\|H-I\|$. Obviously, $K_{1}^{2}(\|H-I\|)^{2} \leq V(n, H, I) \leq K_{2}^{2}(\| H-$ $I \|)^{2}$.

Let $\alpha(x)=K_{1}^{2} x^{2}$ and $\beta(x)=K_{2}^{2} x^{2}$. Then condition (1) of Lemma 4 is satisfied. Furthermore, for any 
$(n, H, I),(n, \widehat{H}, \widehat{I}) \in \mathscr{Z}^{+} \times \Gamma \times \Gamma$, we replace $\left(u_{i}(n)-w_{i}(n)\right)$ and $\left(\widehat{u}_{i}(n)-\widehat{w}_{i}(n)\right), i=1,2$ with $\mathbb{D}_{i}(n)$ and $\widehat{\mathbb{D}}_{i}(n)$, respectively; we have

$$
\begin{aligned}
&|V(n, H, I)-V(n, \widehat{H}, \widehat{I})| \\
&=\left|\mathbb{D}_{1}^{2}(n)+\mathbb{D}_{2}^{2}(n)-\widehat{\mathbb{D}}_{1}^{2}(n)-\widehat{\mathbb{D}}_{2}^{2}(n)\right| \\
& \leq\left|\mathbb{D}_{1}^{2}(n)-\widehat{\mathbb{D}}_{1}^{2}(n)\right|+\left|\mathbb{D}_{2}^{2}(n)-\widehat{\mathbb{D}}_{2}^{2}(n)\right| \\
&=\left|\mathbb{D}_{1}(n)+\widehat{\mathbb{D}}_{1}(n)\right|\left|\mathbb{D}_{1}(n)-\widehat{\mathbb{D}}_{1}(n)\right| \\
& \quad+\left|\mathbb{D}_{2}(n)+\widehat{\mathbb{D}}_{2}(n)\right|\left|\mathbb{D}_{2}(n)-\widehat{\mathbb{D}}_{2}(n)\right| \\
& \leq\left(\left|u_{1}(n)\right|+\left|w_{1}(n)\right|+\left|\widehat{u}_{1}(n)\right|+\left|\widehat{w}_{1}(n)\right|\right) \\
& \cdot\left(\left|u_{1}(n)-\widehat{u}_{1}(n)\right|+\left|w_{1}-\widehat{w}_{1}(n)\right|\right) \\
&+\left(\left|u_{2}(n)\right|+\left|w_{2}(n)\right|+\left|\widehat{u}_{2}(n)\right|+\left|\widehat{w}_{2}(n)\right|\right) \\
& \cdot\left(\left|u_{2}(n)-\widehat{u}_{2}(n)\right|+\left|w_{2}-\widehat{w}_{2}(n)\right|\right) \\
& \leq G\left\{\left|u_{1}(n)-\widehat{u}_{1}(n)\right|+\left|u_{2}(n)-\widehat{u}_{2}(n)\right|\right. \\
&\left.+\left|w_{1}-\widehat{w}_{1}(n)\right|+\left|w_{2}-\widehat{w}_{2}(n)\right|\right\} \\
&= G\left\{|| H-\widehat{H}\|+\| I-\widehat{I}_{\|} \|\right\},
\end{aligned}
$$

where $\widehat{H}=\left(\widehat{u}_{1}(n), \widehat{u}_{2}(n)\right), \widehat{I}=\left(\widehat{w}_{1}(n), \widehat{w}_{2}(n)\right)$, and $G=$ $4 \max \left\{s_{1}, s_{2}\right\}$. Consequently, condition (2) of Lemma 4 is satisfied. At last, calculating the $\Delta V(n)$ of $V(n)$ along the solutions of system (34) yields

$$
\begin{aligned}
\Delta & V_{(34)}(n) \\
= & V(n+1)-V(n) \\
= & \mathbb{D}_{1}^{2}(n+1)+\mathbb{D}_{2}^{2}(n+1)-\mathbb{D}_{1}^{2}(n)-\mathbb{D}_{2}^{2}(n) \\
= & {\left[\mathbb{D}_{1}^{2}(n+1)-\mathbb{D}_{1}(n)^{2}\right] } \\
& +\left[\mathbb{D}_{2}^{2}(n+1)-\mathbb{D}_{2}(n)^{2}\right] \\
= & {\left[\mathbb{D}_{1}(n)-b_{1}(n)\left(e^{u_{1}(n)}-e^{w_{1}(n)}\right)\right.} \\
& \left.-\left(\frac{a_{2}(n) e^{u_{2}(n)}}{1+d_{1}(n) e^{u_{1}(n)}}-\frac{a_{2}(n) e^{w_{2}(n)}}{1+d_{1}(n) e^{w_{1}(n)}}\right)\right]^{2}-\mathbb{D}_{1}(n)^{2} \\
& +\left[\mathbb{D}_{2}(n)-b_{2}(n)\left(e^{u_{2}(n)}-e^{w_{2}(n)}\right)\right. \\
& \left.\quad-\left(\frac{a_{1}(n) e^{u_{1}(n)}}{1+d_{2}(n) e^{u_{2}(n)}}-\frac{a_{1}(n) e^{w_{1}(n)}}{1+d_{2}(n) e^{w_{2}(n)}}\right)\right]^{2}-\mathbb{D}_{2}(n)^{2} \\
= & -2 b_{1}(n) \mathbb{D}_{1}(n)\left(e^{u_{1}(n)}-e^{w_{1}(n)}\right) \\
& -2 a_{2}(n) \mathbb{D}_{1}(n) \\
& \cdot\left(\frac{e^{u_{2}(n)}}{1+d_{1}(n) e^{u_{1}(n)}}-\frac{e^{w_{2}(n)}}{1+d_{1}(n) e^{w_{1}(n)}}\right)
\end{aligned}
$$

$$
\begin{aligned}
& +2 a_{2}(n) b_{1}(n)\left(e^{u_{1}(n)}-e^{w_{1}(n)}\right) \\
& \cdot\left(\frac{e^{u_{2}(n)}}{1+d_{1}(n) e^{u_{1}(n)}}-\frac{e^{w_{2}(n)}}{1+d_{1}(n) e^{w_{1}(n)}}\right) \\
& +b_{1}^{2}(n)\left(e^{u_{1}(n)}-e^{w_{1}(n)}\right)^{2} \\
& +a_{2}^{2}(n)\left(\frac{e^{u_{2}(n)}}{1+d_{1}(n) e^{u_{1}(n)}}-\frac{e^{w_{2}(n)}}{1+d_{1}(n) e^{w_{1}(n)}}\right)^{2} \\
& -2 b_{2}(n) \mathbb{D}_{2}(n)\left(e^{u_{2}(n)}-e^{w_{2}(n)}\right) \\
& -2 a_{1}(n) \mathbb{D}_{2}(n) \\
& \cdot\left(\frac{e^{u_{1}(n)}}{1+d_{2}(n) e^{u_{2}(n)}}-\frac{e^{w_{1}(n)}}{1+d_{2}(n) e^{w_{2}(n)}}\right) \\
& +2 a_{1}(n) b_{2}(n)\left(e^{u_{2}(n)}-e^{w_{2}(n)}\right) \\
& \cdot\left(\frac{e^{u_{1}(n)}}{1+d_{2}(n) e^{u_{2}(n)}}-\frac{e^{w_{1}(n)}}{1+d_{2}(n) e^{w_{2}(n)}}\right) \\
& +b_{2}^{2}(n)\left(e^{u_{2}(n)}-e^{w_{2}(n)}\right)^{2} \\
& +a_{1}^{2}(n)\left(\frac{e^{u_{1}(n)}}{1+d_{2}(n) e^{u_{2}(n)}}-\frac{e^{w_{1}(n)}}{1+d_{2}(n) e^{w_{2}(n)}}\right)^{2} .
\end{aligned}
$$

Applying the one-dimensional and two-dimensional mean value theorem, we arrive at a simple result as follows:

$$
\begin{gathered}
e^{u_{i}(n)}-e^{w_{i}(n)}=\zeta_{i}(n)\left(u_{i}(n)-w_{i}(n)\right), \\
\frac{e^{u_{i}(n)}}{1+d_{j}(n) e^{u_{j}(n)}}-\frac{e^{w_{i}(n)}}{1+d_{j}(n) e^{w_{j}(n)}} \\
=\frac{\theta_{i}(n)}{1+d_{j}(n) \theta_{j}(n)}\left(u_{i}(n)-w_{i}(n)\right) \\
\quad-\frac{d_{j}(n) \theta_{i}(n) \theta_{j}(n)}{\left(1+d_{j}(n) \theta_{j}(n)\right)^{2}}\left(u_{j}(n)-w_{j}(n)\right),
\end{gathered}
$$

where $i, j=1,2, i \neq j$, and $\zeta_{i}(n)$ and $\theta_{i}(n)$ lie between $e^{u_{i}(n)}$ and $e^{w_{i}(n)}$, respectively. Substituting (38) into (37), one obtains

$$
\begin{aligned}
\Delta & V_{(34)}(n) \\
= & -2 b_{1}(n) \zeta_{1}(n) \mathbb{D}_{1}^{2}(n) \\
& +\frac{2 a_{2}(n) d_{1}(n) \theta_{1}(n) \theta_{2}(n)}{\left(1+d_{1}(n) \theta_{1}(n)\right)^{2}} \mathbb{D}_{1}^{2}(n) \\
& -\frac{2 a_{2}(n) \theta_{2}(n)}{1+d_{1}(n) \theta_{1}(n)} \mathbb{D}_{1}(n) \mathbb{D}_{2}(n)
\end{aligned}
$$




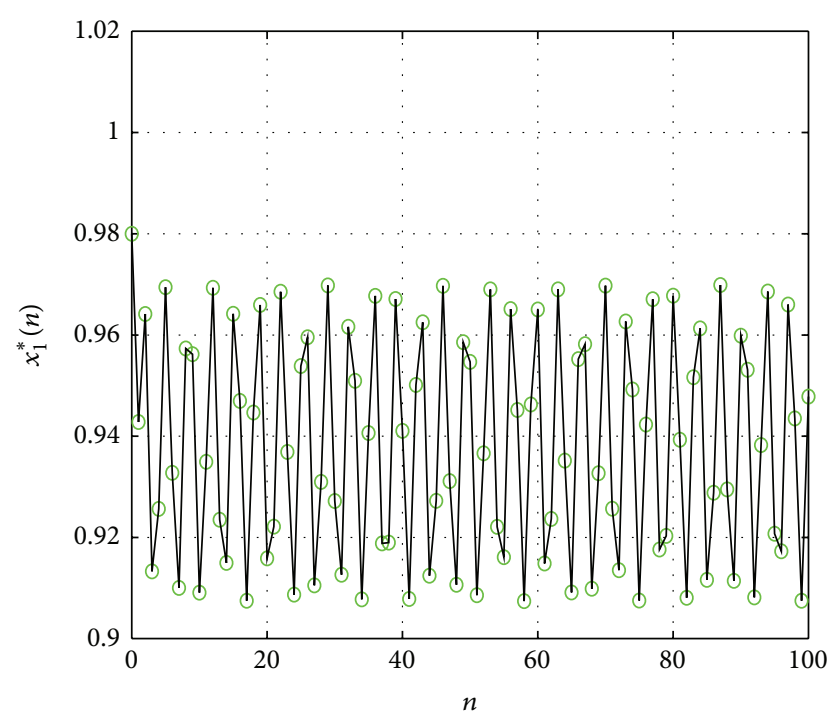

(a)

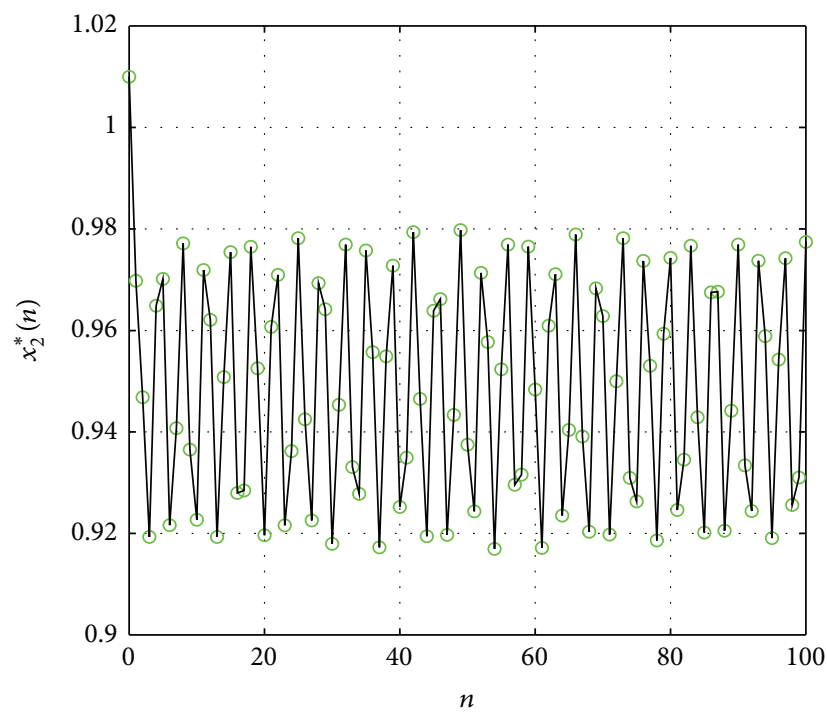

(c)

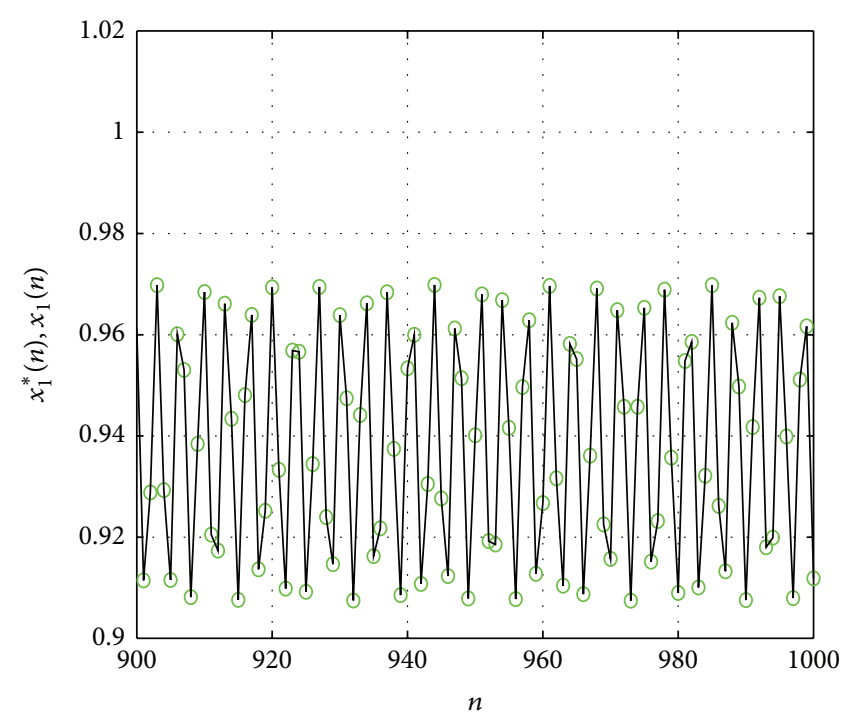

(b)

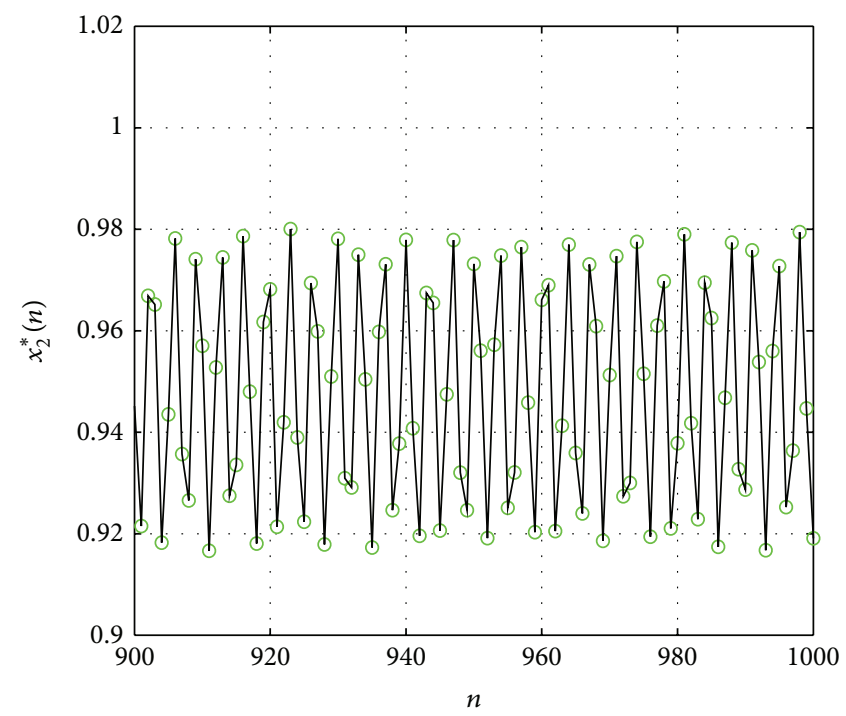

(d)

Figure 1: Positive almost periodic solution of system (22). (a) and (c) Time-series $x_{1}^{*}(n)$ and $x_{2}^{*}(n)$ with initial values $x_{1}^{*}(0)=0.98$ and $x_{2}^{*}(0)=1.01$, for $n \in[0,100]$, and $(\mathrm{b})$ and $(\mathrm{d})$ show that $x_{1}^{*}(n)$ and $x_{2}^{*}(n)$ have the same initial data for $n \in[900$, 1000], respectively.

$$
\begin{array}{cc}
+a_{2}^{2}(n)\left[\frac{\left(d_{1}(n) \theta_{1}(n) \theta_{2}(n)\right)^{2}}{\left(1+d_{1}(n) \theta_{1}(n)\right)^{4}} \mathbb{D}_{1}^{2}(n)\right. & +b_{1}^{2}(n) \zeta_{1}^{2}(n) \mathbb{D}_{1}^{2}(n)-2 b_{2}(n) \zeta_{2}(n) \mathbb{D}_{2}^{2}(n) \\
+\frac{\theta_{2}^{2}(n)}{\left(1+d_{1}(n) \theta_{1}(n)\right)^{2}} \mathbb{D}_{2}^{2}(n) & +\frac{2 a_{1}(n) d_{2}(n) \theta_{1}(n) \theta_{2}(n)}{\left(1+d_{2}(n) \theta_{2}(n)\right)^{2}} \mathbb{D}_{2}^{2}(n) \\
\left.-\frac{2 d_{1}(n) \theta_{1}(n) \theta_{2}^{2}(n)}{\left(1+d_{1}(n) \theta_{1}(n)\right)^{3}} \mathbb{D}_{1}(n) \mathbb{D}_{2}(n)\right] & -\frac{2 a_{1}(n) \theta_{1}(n)}{1+d_{2}(n) \theta_{2}(n)} \mathbb{D}_{1}(n) \mathbb{D}_{2}(n) \\
-\frac{2 a_{2}(n) b_{1}(n) d_{1}(n) \zeta_{1}(n) \theta_{1}(n) \theta_{2}(n)}{\left(1+d_{1}(n) \theta_{1}(n)\right)^{2}} \mathbb{D}_{1}^{2}(n) & +a_{1}^{2}(n)\left[\frac{\left(d_{2}(n) \theta_{1}(n) \theta_{2}(n)\right)^{2}}{\left(1+d_{2}(n) \theta_{2}(n)\right)^{4}} \mathbb{D}_{2}^{2}(n)\right. \\
+\frac{2 a_{2}(n) b_{1}(n) \zeta_{1}(n) \theta_{2}(n)}{1+d_{1}(n) \theta_{1}(n)} \mathbb{D}_{1}(n) \mathbb{D}_{2}(n) & +\frac{\theta_{1}^{2}(n)}{\left(1+d_{2}(n) \theta_{2}(n)\right)^{2}} \mathbb{D}_{1}^{2}(n)
\end{array}
$$




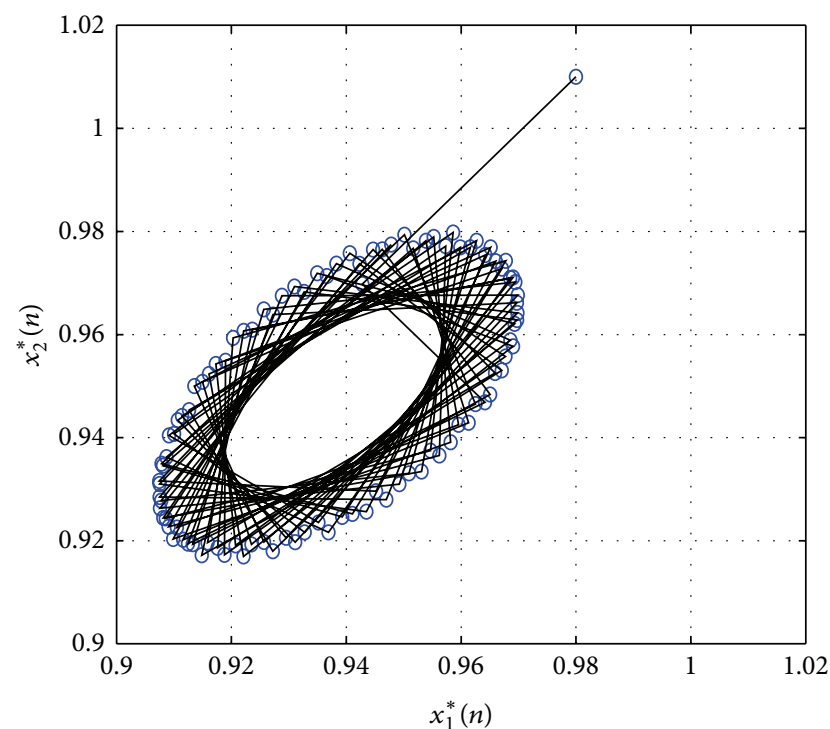

(a)

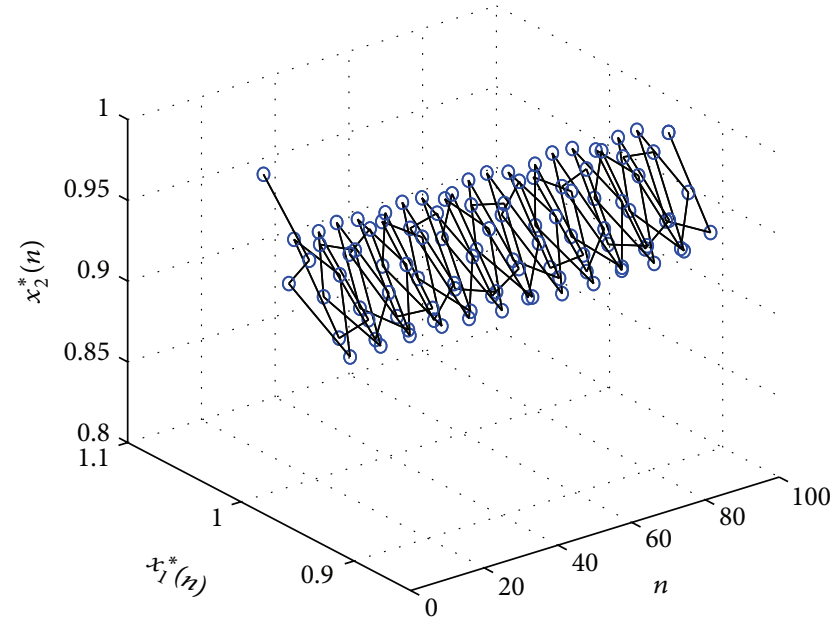

(c)

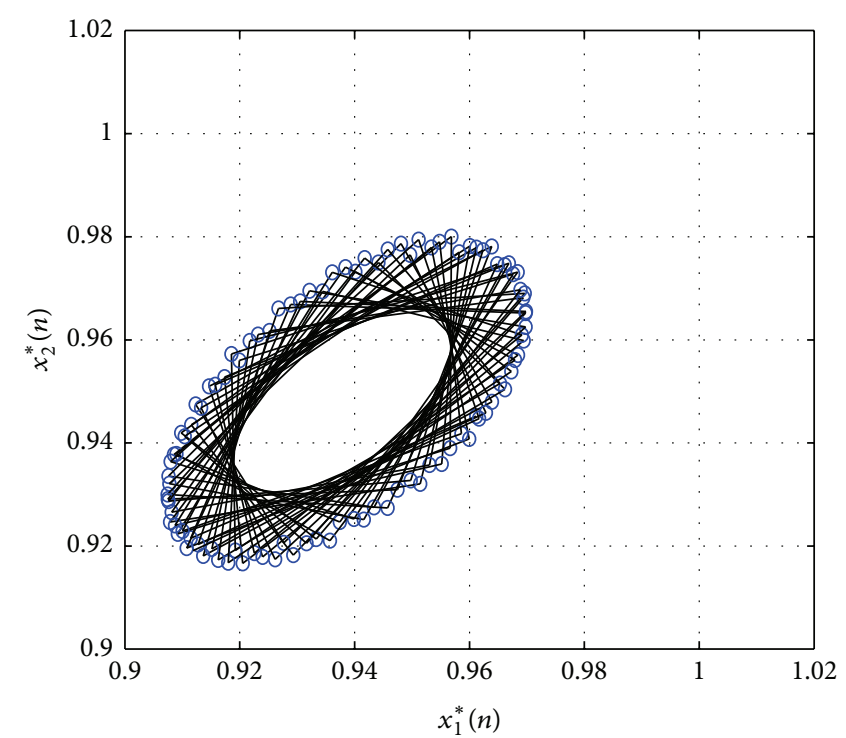

(b)

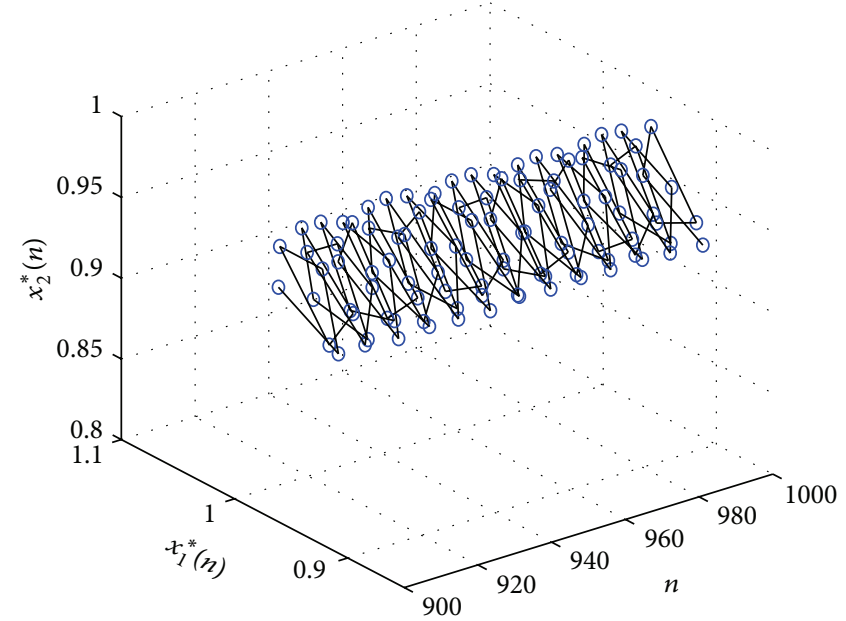

(d)

FIGURE 2: Phase portrait; 2-dimensional and 3-dimensional phase portraits of almost periodic solution of system (22). Time-series $x_{1}^{*}(n)$ and $x_{2}^{*}(n)$ with initial values $x_{1}^{*}(0)=0.98$ and $x_{2}^{*}(0)=1.01$; (a) and (c) indicate $n \in[0,100]$ and (b) and (d) indicate $n \in[900,1000]$, respectively.

$$
\begin{array}{ccc} 
& \left.-\frac{2 d_{2}(n) \theta_{1}(n)^{2}(n) \theta_{2}(n)}{\left(1+d_{2}(n) \theta_{2}(n)\right)^{3}} \mathbb{D}_{1}(n) \mathbb{D}_{2}(n)\right] & +\frac{\left(a_{2}(n) d_{1}(n) \theta_{1}(n) \theta_{2}(n)\right)^{2}}{\left(1+d_{1}(n) \theta_{1}(n)\right)^{4}}+\frac{\left(a_{1}(n) \theta_{1}(n)\right)^{2}}{\left(1+d_{2}(n) \theta_{2}(n)\right)^{2}} \\
-\frac{2 a_{1}(n) b_{2}(n) d_{2}(n) \zeta_{2}(n) \theta_{1}(n) \theta_{2}(n)}{\left(1+d_{2}(n) \theta_{2}(n)\right)^{2}} \mathbb{D}_{2}^{2}(n) & \left.-\frac{2 a_{2}(n) b_{1}(n) d_{1}(n) \zeta_{1}(n) \theta_{1}(n) \theta_{2}(n)}{\left(1+d_{1}(n) \theta_{1}(n)\right)^{2}}\right] \mathbb{D}_{1}^{2}(n) \\
+\frac{2 a_{1}(n) b_{2}(n) \zeta_{2}(n) \theta_{1}(n)}{1+d_{2}(n) \theta_{2}(n)} \mathbb{D}_{1}(n) \mathbb{D}_{2}(n) & +\left[-2 b_{2}(n) \zeta_{2}(n)+\frac{2 a_{1}(n) d_{2}(n) \theta_{1}(n) \theta_{2}(n)}{\left(1+d_{2}(n) \theta_{2}(n)\right)^{2}}\right. \\
+b_{2}^{2}(n) \zeta_{2}^{2}(n) \mathbb{D}_{2}^{2}(n) & +b_{2}^{2}(n) \zeta_{2}^{2}(n)+\frac{\left(a_{1}(n) d_{2}(n) \theta_{1}(n) \theta_{2}(n)\right)^{2}}{\left(1+d_{2}(n) \theta_{2}(n)\right)^{4}} \\
=\left[\begin{array}{l}
-2 b_{1}(n) \zeta_{1}(n)+\frac{2 a_{2}(n) d_{1}(n) \theta_{1}(n) \theta_{2}(n)}{\left(1+d_{1}(n) \theta_{1}(n)\right)^{2}} \\
+b_{1}^{2}(n) \zeta_{1}^{2}(n)
\end{array}\right. & -\frac{2 a_{1}(n) b_{2}(n) d_{2}(n) \zeta_{2}(n) \theta_{1}(n) \theta_{2}(n)}{\left(1+d_{2}(n) \theta_{2}(n)\right)^{2}}
\end{array}
$$



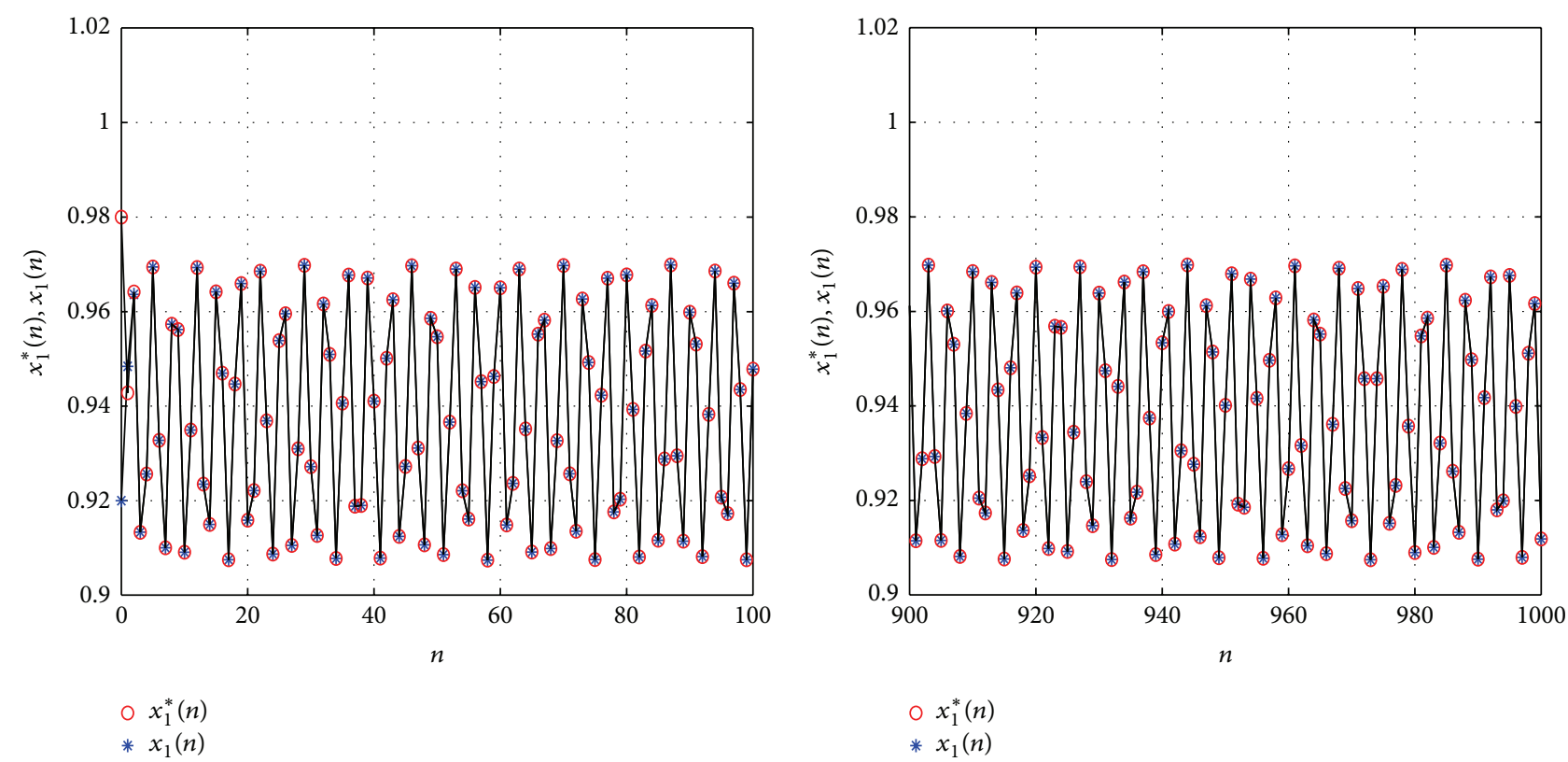

(a)
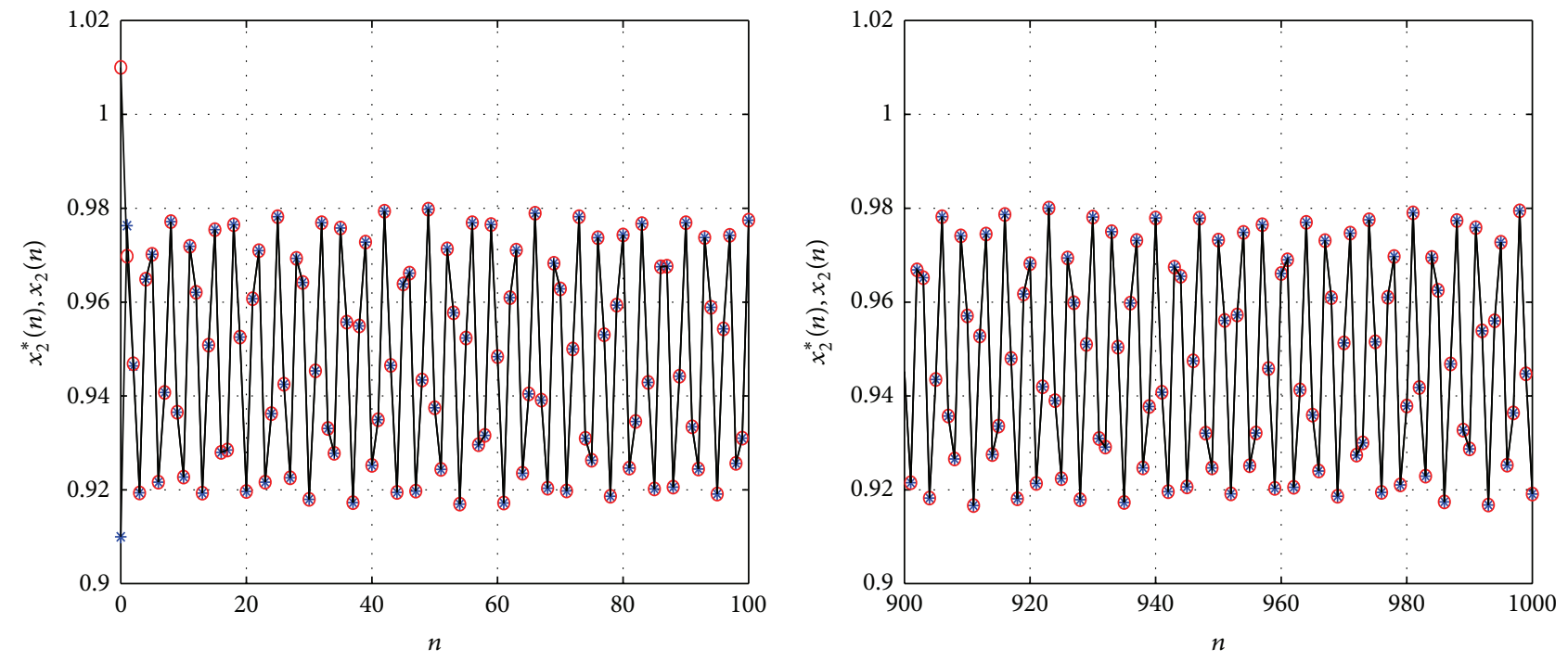

$\begin{array}{ll}\circ & x_{2}^{*}(n) \\ * & x_{2}(n)\end{array}$

O $x_{2}^{*}(n)$

* $x_{2}(n)$

(c)

(d)

FIGURE 3: Uniformly asymptotic stability. Time-series $x_{1}^{*}(n)$ and $x_{2}^{*}(n)$ with initial values $x_{1}^{*}(0)=0.98$ and $x_{2}^{*}(0)=1.01$ and $x_{1}(n)$ and $x_{2}(n)$ with initial values $x_{1}(0)=0.92$ and $x_{2}(0)=0.91$. (a) and (c) indicate $n \in[0,100]$ and (b) and (d) indicate $n \in[900,1000]$, respectively.

$$
\begin{array}{cc}
\left.+\frac{\left(a_{2}(n) \theta_{2}(n)\right)^{2}}{\left(1+d_{1}(n) \theta_{1}(n)\right)^{2}}\right] \mathbb{D}_{2}^{2}(n) & -\frac{2 a_{1}^{2}(n) d_{2}(n) \theta_{2}(n) \theta_{1}^{2}(n)}{\left(1+d_{2}(n) \theta_{2}(n)\right)^{3}} \\
+\left[-\frac{2 a_{2}(n) \theta_{2}(n)}{1+d_{1}(n) \theta_{1}(n)}-\frac{2 a_{2}^{2}(n) d_{1}(n) \theta_{1}(n) \theta_{2}^{2}(n)}{\left(1+d_{1}(n) \theta_{1}(n)\right)^{3}}\right. & \left.+\frac{2 a_{1}(n) b_{2}(n) \zeta_{2}(n) \theta_{1}(n)}{1+d_{2}(n) \theta_{2}(n)}\right] \mathbb{D}_{1}(n) \mathbb{D}_{2}(n) \\
+\frac{2 a_{2}(n) b_{1}(n) \zeta_{1}(n) \theta_{2}(n)}{1+d_{1}(n) \theta_{1}(n)}-\frac{2 a_{1}(n) \theta_{1}(n)}{1+d_{2}(n) \theta_{2}(n)} & \leq\left[-2 b_{1}(n) \zeta_{1}(n)+\frac{2 a_{2}(n) d_{1}(n) \theta_{1}(n) \theta_{2}(n)}{\left(1+d_{1}(n) \theta_{1}(n)\right)^{2}}\right.
\end{array}
$$




$$
\begin{aligned}
& +b_{1}^{2}(n) \zeta_{1}^{2}(n)+\frac{\left(a_{2}(n) d_{1}(n) \theta_{1}(n) \theta_{2}(n)\right)^{2}}{\left(1+d_{1}(n) \theta_{1}(n)\right)^{4}} \\
& -\frac{2 a_{2}(n) b_{1}(n) d_{1}(n) \zeta_{1}(n) \theta_{1}(n) \theta_{2}(n)}{\left(1+d_{1}(n) \theta_{1}(n)\right)^{2}} \\
& +\frac{\left(a_{1}(n) \theta_{1}(n)\right)^{2}}{\left(1+d_{2}(n) \theta_{2}(n)\right)^{2}}+\frac{a_{2}(n) \theta_{2}(n)}{1+d_{1}(n) \theta_{1}(n)} \\
& +\frac{a_{2}^{2}(n) d_{1}(n) \theta_{1}(n) \theta_{2}^{2}(n)}{\left(1+d_{1}(n) \theta_{1}(n)\right)^{3}} \\
& +\frac{a_{2}(n) b_{1}(n) \zeta_{1}(n) \theta_{2}(n)}{1+d_{1}(n) \theta_{1}(n)}+\frac{a_{1}(n) \theta_{1}(n)}{1+d_{2}(n) \theta_{2}(n)} \\
& +\frac{a_{1}^{2}(n) d_{2}(n) \theta_{2}(n) \theta_{1}^{2}(n)}{\left(1+d_{2}(n) \theta_{2}(n)\right)^{3}} \\
& \left.+\frac{a_{1}(n) b_{2}(n) \zeta_{2}(n) \theta_{1}(n)}{1+d_{2}(n) \theta_{2}(n)}\right] \mathbb{D}_{1}^{2}(n) \\
& +\left[-2 b_{2}(n) \zeta_{2}(n)+\frac{2 a_{1}(n) d_{2}(n) \theta_{1}(n) \theta_{2}(n)}{\left(1+d_{2}(n) \theta_{2}(n)\right)^{2}}\right. \\
& +b_{2}^{2}(n) \zeta_{2}^{2}(n)+\frac{\left(a_{1}(n) d_{2}(n) \theta_{1}(n) \theta_{2}(n)\right)^{2}}{\left(1+d_{2}(n) \theta_{2}(n)\right)^{4}} \\
& -\frac{2 a_{1}(n) b_{2}(n) d_{2}(n) \zeta_{2}(n) \theta_{1}(n) \theta_{2}(n)}{2} \\
& \left(1+d_{2}(n) \theta_{2}(n)\right)^{2} \\
& +\frac{\left(a_{2}(n) \theta_{2}(n)\right)^{2}}{\left(1+d_{1}(n) \theta_{1}(n)\right)^{2}}+\frac{a_{2}(n) \theta_{2}(n)}{1+d_{1}(n) \theta_{1}(n)} \\
& +\frac{a_{2}^{2}(n) d_{1}(n) \theta_{1}(n) \theta_{2}^{2}(n)}{\left(1+d_{1}(n) \theta_{1}(n)\right)^{3}}+\frac{a_{2}(n) b_{1}(n) \zeta_{1}(n) \theta_{2}(n)}{1+d_{1}(n) \theta_{1}(n)} \\
& +\frac{a_{1}(n) \theta_{1}(n)}{1+d_{2}(n) \theta_{2}(n)}+\frac{a_{1}^{2}(n) d_{2}(n) \theta_{2}(n) \theta_{1}^{2}(n)}{\left(1+d_{2}(n) \theta_{2}(n)\right)^{3}} \\
& \left.+\frac{a_{1}(n) b_{2}(n) \zeta_{2}(n) \theta_{1}(n)}{1+d_{2}(n) \theta_{2}(n)}\right] \mathbb{D}_{2}^{2}(n) \\
& \leq\left[-2 b_{1}^{l} m_{1}+\frac{a_{2}^{u} M_{2}+a_{2}^{u} b_{1}^{u} M_{1} M_{2}}{1+d_{1}^{l} m_{1}}\right. \\
& +\frac{a_{1}^{u} M_{1}+a_{1}^{u} b_{2}^{u} M_{1} M_{2}}{1+d_{2}^{l} m_{2}}+\left(b_{1}^{u} M_{1}\right)^{2} \\
& +\frac{2\left(a_{2}^{u} d_{1}^{u} M_{1} M_{2}-a_{2}^{l} b_{1}^{l} d_{1}^{l} m_{1}^{2} m_{2}\right)}{\left(1+d_{1}^{l} m_{1}\right)^{2}} \\
& +\frac{\left(a_{1}^{u} M_{1}\right)^{2}}{\left(1+d_{2}^{l} m_{2}\right)^{2}}+\frac{a_{2}^{u 2} d_{1}^{u} M_{1} M_{2}^{2}}{\left(1+d_{1}^{l} m_{1}\right)^{3}}
\end{aligned}
$$

$$
\begin{aligned}
& \left.+\frac{a_{1}^{u 2} d_{2}^{u} M_{1}^{2} M_{2}}{\left(1+d_{2}^{l} m_{2}\right)^{3}}+\frac{\left(a_{2}^{u} d_{1}^{u} M_{1} M_{2}\right)^{2}}{\left(1+d_{1}^{l} m_{1}\right)^{4}}\right] \mathbb{D}_{1}^{2}(n) \\
& +\left[-2 b_{2}^{l} m_{2}+\frac{a_{2}^{u} M_{2}+a_{2}^{u} b_{1}^{u} M_{1} M_{2}}{1+d_{1}^{l} m_{1}}\right. \\
& +\frac{a_{1}^{u} M_{1}+a_{1}^{u} b_{2}^{u} M_{1} M_{2}}{1+d_{2}^{l} m_{2}}+\left(b_{2}^{u} M_{2}\right)^{2} \\
& +\frac{2\left(a_{1}^{u} d_{2}^{u} M_{1} M_{2}-a_{2}^{l} b_{2}^{l} d_{2}^{l} m_{1} m_{2}^{2}\right)}{\left(1+d_{2}^{l} m_{2}\right)^{2}} \\
& +\frac{\left(a_{2}^{u} M_{2}\right)^{2}}{\left(1+d_{1}^{l} m_{1}\right)^{2}}+\frac{a_{2}^{u 2} d_{1}^{u} M_{1} M_{2}^{2}}{\left(1+d_{1}^{l} m_{1}\right)^{3}} \\
& \left.+\frac{a_{1}^{u 2} d_{2}^{u} M_{1}^{2} M_{2}}{\left(1+d_{2}^{l} m_{2}\right)^{3}}+\frac{\left(a_{1}^{u} d_{2}^{u} M_{1} M_{2}\right)^{2}}{\left(1+d_{2}^{l} m_{2}\right)^{4}}\right] \mathbb{D}_{2}^{2}(n) \\
& =-\left[2 b_{1}^{l} m_{1}-\frac{a_{2}^{u} M_{2}+a_{2}^{u} b_{1}^{u} M_{1} M_{2}}{1+d_{1}^{l} m_{1}}\right. \\
& -\frac{a_{1}^{u} M_{1}+a_{1}^{u} b_{2}^{u} M_{1} M_{2}}{1+d_{2}^{l} m_{2}}-\left(b_{1}^{u} M_{1}\right)^{2} \\
& -\frac{2\left(a_{2}^{u} d_{1}^{u} M_{1} M_{2}-a_{2}^{l} b_{1}^{l} d_{1}^{l} m_{1}^{2} m_{2}\right)}{\left(1+d_{1}^{l} m_{1}\right)^{2}} \\
& -\frac{\left(a_{1}^{u} M_{1}\right)^{2}}{\left(1+d_{2}^{l} m_{2}\right)^{2}}-\frac{a_{2}^{u 2} d_{1}^{u} M_{1} M_{2}^{2}}{\left(1+d_{1}^{l} m_{1}\right)^{3}} \\
& \left.-\frac{a_{1}^{u 2} d_{2}^{u} M_{1}^{2} M_{2}}{\left(1+d_{2}^{l} m_{2}\right)^{3}}-\frac{\left(a_{2}^{u} d_{1}^{u} M_{1} M_{2}\right)^{2}}{\left(1+d_{1}^{l} m_{1}\right)^{4}}\right] \mathbb{D}_{1}^{2}(n) \\
& -\left[2 b_{2}^{l} m_{2}-\frac{a_{2}^{u} M_{2}+a_{2}^{u} b_{1}^{u} M_{1} M_{2}}{1+d_{1}^{l} m_{1}}\right. \\
& -\frac{a_{1}^{u} M_{1}+a_{1}^{u} b_{2}^{u} M_{1} M_{2}}{1+d_{2}^{l} m_{2}}-\left(b_{2}^{u} M_{2}\right)^{2} \\
& -\frac{2\left(a_{1}^{u} d_{2}^{u} M_{1} M_{2}-a_{2}^{l} b_{2}^{l} d_{2}^{l} m_{1} m_{2}^{2}\right)}{\left(1+d_{2}^{l} m_{2}\right)^{2}} \\
& -\frac{\left(a_{2}^{u} M_{2}\right)^{2}}{\left(1+d_{1}^{l} m_{1}\right)^{2}}-\frac{a_{2}^{u 2} d_{1}^{u} M_{1} M_{2}^{2}}{\left(1+d_{1}^{l} m_{1}\right)^{3}} \\
& \left.-\frac{a_{1}^{u 2} d_{2}^{u} M_{1}^{2} M_{2}}{\left(1+d_{2}^{l} m_{2}\right)^{3}}-\frac{\left(a_{1}^{u} d_{2}^{u} M_{1} M_{2}\right)^{2}}{\left(1+d_{2}^{l} m_{2}\right)^{4}}\right] \mathbb{D}_{2}^{2}(n) \\
& =-\left[\beta_{1} \mathbb{D}_{1}^{2}(n)+\beta_{2} \mathbb{D}_{2}^{2}(n)\right] \\
& =-\gamma\left[\mathbb{D}_{1}^{2}(n)+\mathbb{D}_{2}^{2}(n)\right]=-\gamma V_{n} \text {, }
\end{aligned}
$$


where $\gamma=\min \left\{\beta_{1}, \beta_{2}\right\}$ and $0<\gamma<1$ which has been pointed out in Theorem 11. In addition, condition (3) of Lemma 4 is also satisfied. According to Lemma 4, there exists a uniformly asymptotically stable almost periodic solution $\left(u_{1}^{*}(n), u_{2}^{*}(n)\right)$ of system (31) which is bounded by $\Gamma$ for all $n \in \mathscr{Z}^{+}$. Namely, there exists a uniformly asymptotically stable almost periodic solution $\left(x_{1}^{*}(n), x_{2}^{*}(n)\right)$ of system (2) which is bounded by $\Omega$ for all $n \in \mathscr{Z}^{+}$. This completed the proof.

\section{Example and Numerical Simulations}

In this section, we only give the following example about almost periodic solutions to check the feasibility of the assumptions of Theorem 11 considering that the simulation about periodic model is similar.

Example 1. Consider the following discrete system:

$$
\begin{aligned}
& x_{1}(n+1) \\
& =x_{1}(n) \exp [1.09-0.03 \sin (\sqrt{2} n \pi) \\
& -(1.15-0.01 \cos (\sqrt{2} n \pi)) x_{1}(n) \\
& \left.-\frac{(0.035+0.005 \cos (\sqrt{2} n \pi)) x_{2}(n)}{1+(2.10+0.02 \cos (\sqrt{5} n \pi)) x_{1}(n)}\right] \text {, } \\
& x_{2}(n+1) \\
& =x_{2}(n) \exp [1.06+0.03 \cos (\sqrt{2} n \pi) \\
& -(1.11+0.01 \sin (\sqrt{2} n \pi)) x_{2}(n) \\
& \left.-\frac{(0.025+0.005 \cos (\sqrt{2} n \pi)) x_{1}(n)}{1+(2.07+0.03 \sin (\sqrt{5} n \pi)) x_{2}(n)}\right] \text {, }
\end{aligned}
$$

with the following intial conditions:

$$
\begin{aligned}
& x_{1}(n)^{*}(0)=0.98 \\
& x_{2}(n)^{*}(0)=1.01 .
\end{aligned}
$$

By a computation, we get

$$
\begin{aligned}
M_{1} & \approx 0.9890, \\
M_{2} & \approx 0.9947, \\
m_{1} & \approx 0.7745, \\
m_{2} & \approx 0.7971, \\
\beta_{1} & \approx 0.3781, \\
\beta_{2} & \approx 0.4438, \\
\left(r_{1}^{l}-a_{2}^{u} M_{2}\right) & \approx 1.0202>0,
\end{aligned}
$$

$$
\begin{aligned}
\left(r_{2}^{l}-a_{1}^{u} M_{1}\right) & \approx 1.0003>0, \\
\frac{b_{1}^{u} M_{1}}{r_{1}^{l}-a_{2}^{u} M_{2}} & \approx 1.1245>1, \\
\frac{b_{2}^{u} M_{2}}{r_{2}^{l}-a_{2}^{u} M_{2}} & \approx 1.1251>1 .
\end{aligned}
$$

Clearly, the assumptions of Theorem 11 are satisfied and all the coefficients are appropriate. Hence, system (40) admits a unique uniformly asymptotically stable positive almost periodic solution. From Figure 1, we easily see that there exists a positive almost periodic solution $\left(x_{1}^{*}(n), x_{2}^{*}(n)\right)$, and the 2-dimensional and 3-dimensional phase portraits of almost periodic system (40) are revealed in Figure 2, respectively. Figure 3 shows that any positive solution $\left(x_{1}(n), x_{2}(n)\right)$ tends to the almost periodic solution $\left(x_{1}^{*}(n), x_{2}^{*}(n)\right)$.

\section{Conclusions}

In this paper, we consider a discrete two-species competitive model whose periodic solutions and almost periodic solutions are discussed, respectively. By the scale law and meanvalue theorem, a good understanding of the existence and stability of positive periodic solutions is gained. Furthermore, by constructing Lyapunov functions, the conditions on the asymptotic stability of the positive almost periodic solution are established. The assumption in (10) implies that the $r(t)$ should be suitably large.

\section{Conflict of Interests}

The authors declare that there is no conflict of interests regarding the publication of this paper.

\section{Acknowledgment}

The work is supported by the National Natural Science Foundation of China (no. 61261044).

\section{References}

[1] K. Gopalsamy, Stability and Oscillations in Delay differential Equations of Populations Dynamics, Kluwer Academic Publishers, Dordrecht, Netherlands, 1992.

[2] Z. Hu, Z. Teng, and H. Jiang, "Stability analysis in a class of discrete SIRS epidemic models," Nonlinear Analysis: Real World Applications, vol. 13, no. 5, pp. 2017-2033, 2012.

[3] P. Das, D. Mukherjee, and A. K. Sarkar, "Study of an S-I epidemic model with nonlinear incidence rate: discrete and stochastic version," Applied Mathematics and Computation, vol. 218, no. 6, pp. 2509-2515, 2011.

[4] Z. Y. Hu, Z. D. Teng, and L. Zhang, "Stability and bifurcation analysis of a discrete predator-prey model with nonmonotonic functional response," Nonlinear Analysis: Real World Applications, vol. 12, no. 4, pp. 2356-2377, 2011.

[5] S. J. Gao and L. S. Chen, "The effect of seasonal harvesting on a single-species discrete population model with stage structure and birth pulses," Chaos, Solitons \& Fractals, vol. 24, no. 4, pp. 1013-1023, 2005. 
[6] Z. Li, F. D. Chen, and M. X. He, "Almost periodic solutions of a discrete Lotka-Volterra competition system with delays," Nonlinear Analysis. Real World Applications, vol. 12, no. 4, pp. 23442355, 2011.

[7] R. P. Agarwal, Difference Equations and Inequalities: Theory, Methods and Applications, Marcel Dekker, New York, NY, USA, 2000.

[8] Q. Wang and Z. Liu, "Uniformly asymptotic stability of positive almost periodic solutions for a discrete competitive system," Journal of Applied Mathematics, vol. 2013, Article ID 182158, 9 pages, 2013.

[9] Z. K. Huang, X. H. Wang, and F. Gao, “The existence and global attractivity of almost periodic sequence solution of discretetime neural networks," Physics Letters A, vol. 350, no. 3-4, pp. 182-191, 2006.

[10] D. Cheban and C. Mammana, "Invariant manifolds, global attractors and almost periodic solutions of nonautonomous difference equations," Nonlinear Analysis: Theory, Methods \& Applications., vol. 56, no. 4, pp. 465-484, 2004.

[11] M. Fan and K. Wang, "Periodic solutions of a discrete time nonautonomous ratio-dependent predator-prey system," Mathematical and Computer Modelling, vol. 35, no. 9-10, pp. 951-961, 2002.

[12] K. Wang, "Periodic solutions to a delayed predator-prey model with Hassell-Varley type functional response," Nonlinear Analysis. Real World Applications, vol. 12, no. 1, pp. 137-145, 2011.

[13] G. R. Liu and J. R. Yan, "Existence of positive periodic solutions for neutral delay Gause-type predator-prey system," Applied Mathematical Modelling, vol. 35, no. 12, pp. 5741-5750, 2011.

[14] X. Chen and C. Fengde, "Stable periodic solution of a discrete periodic Lotka-Volterra competition system with a feedback control," Applied Mathematics and Computation, vol. 181, no. 2, pp. 1446-1454, 2006.

[15] X. Liu, "A note on the existence of periodic solutions in discrete predator-prey models," Applied Mathematical Modelling, vol. 34, no. 9, pp. 2477-2483, 2010.

[16] W. Qin, Z. Liu, and Y. Chen, "Permanence and global stability of positive periodic solutions of a discrete competitive system," Discrete Dynamics in Nature and Society, vol. 2009, Article ID Art. ID 830537, 13 pages, 2009.

[17] C. Y. He, Almost Periodic Differential Equations, Higher Education Press, Beijing, China, 1992.

[18] Y. K. Li, T. W. Zhang, and Y. Ye, "On the existence and stability of a unique almost periodic sequence solution in discrete predator-prey models with time delays," Applied Mathematical Modelling, vol. 35, no. 11, pp. 5448-5459, 2011.

[19] Y. Li, L. Yang, and W. Q. Wu, "Almost periodic solutions for a class of discrete systems with Allee-effect," Applications of Mathematics, vol. 59, no. 2, pp. 191-203, 2014.

[20] S. N. Zhang, "Existence of almost periodic solutions for difference systems," Annals of Differential Equations, vol. 2, pp. 184206, 2000.

[21] X. Yang, "Uniform persistence and periodic solutions for a discrete predator-prey system with delays," Journal of Mathematical Analysis and Applications, vol. 316, no. 1, pp. 161-177, 2006. 


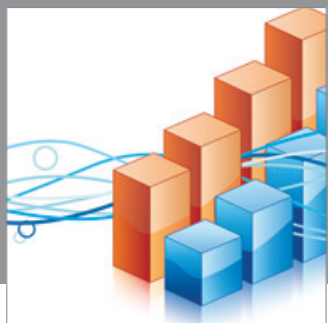

Advances in

Operations Research

mansans

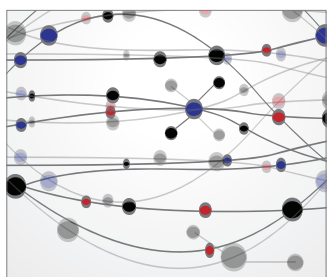

The Scientific World Journal
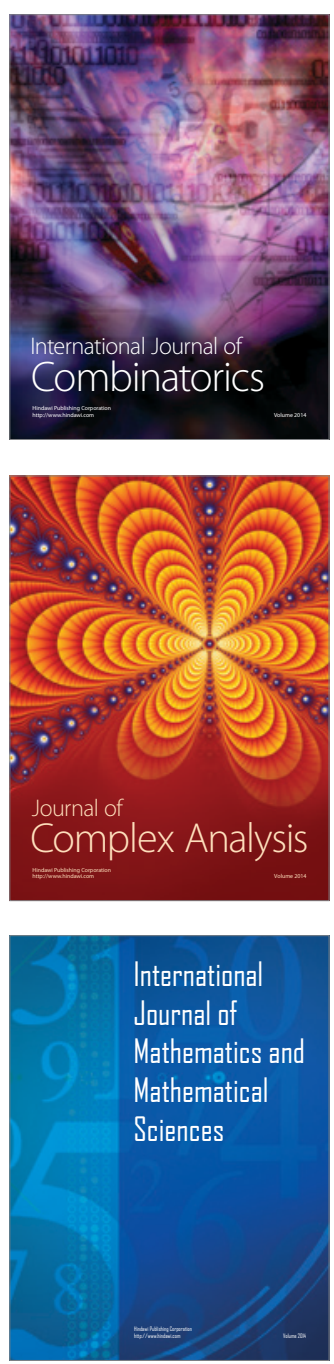
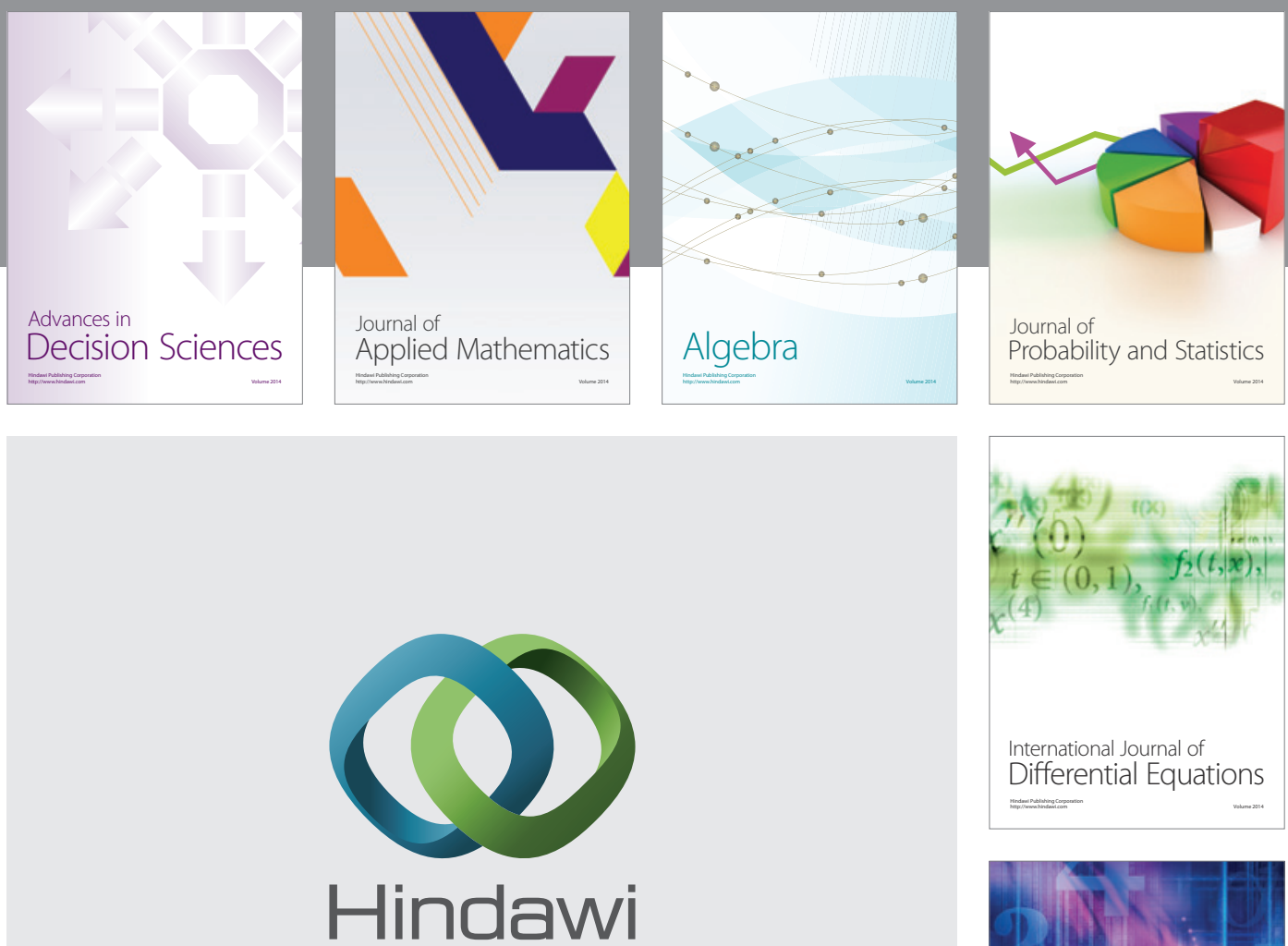

Submit your manuscripts at http://www.hindawi.com
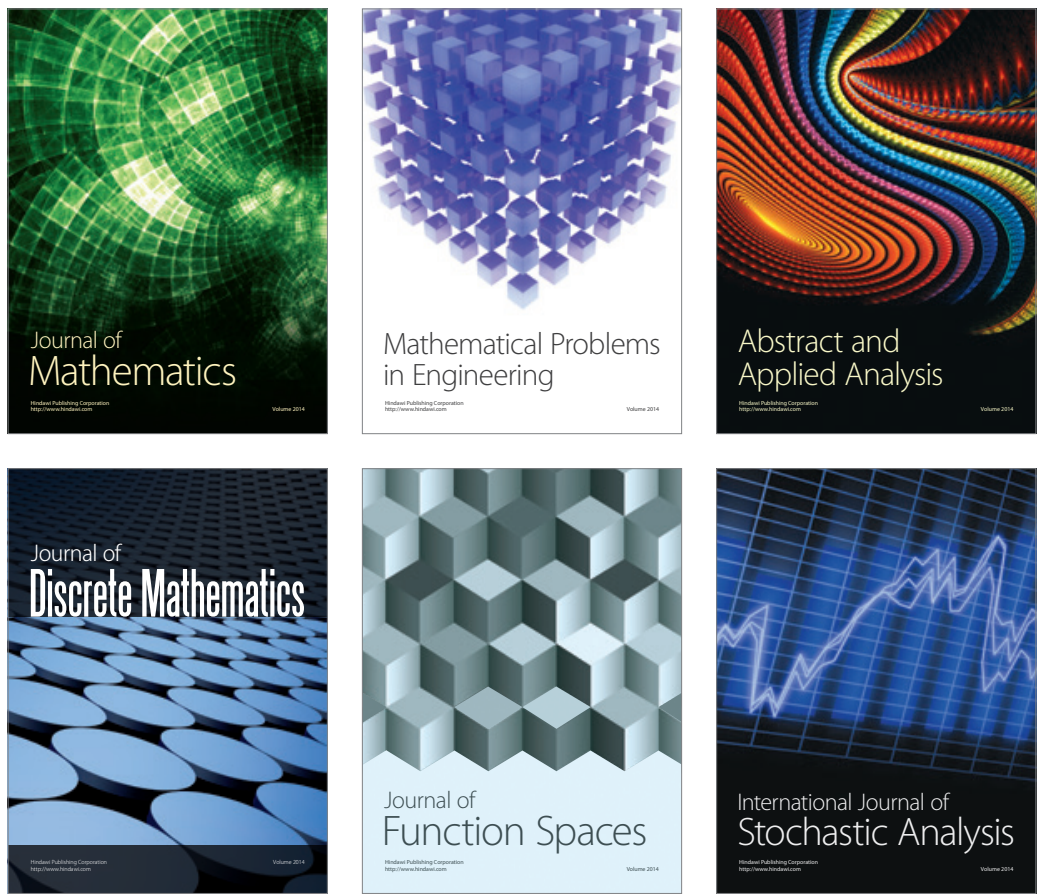

Journal of

Function Spaces

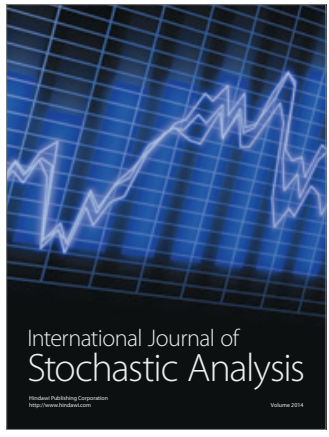

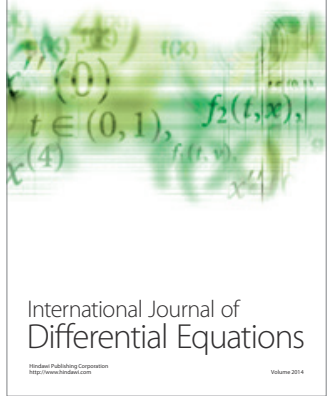
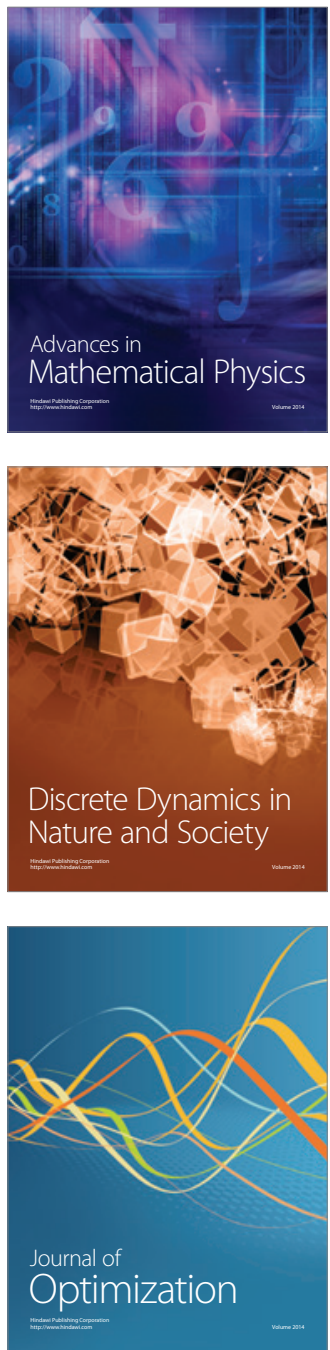